\title{
Hsp70 inhibits aggregation of Islet amyloid polypeptide by binding to the heterogeneous prenucleation oligomers
}

\author{
Neeraja Chilukoti ${ }^{1,2}$, Bankanidhi Sahoo ${ }^{1,3}$, Deepa $\mathrm{S}^{1}$, Sreelakshmi Cherakara ${ }^{1}$, Mithun Maddheshiya ${ }^{1}$ \\ and Kanchan Garai ${ }^{1, *}$ \\ ${ }^{1}$ Tata Institute of Fundamental Research, 36/P Gopanpally, Serilingampally, Hyderabad 500019, India \\ ${ }^{2}$ Current address: Centre for Cellular \& Molecular Biology, Habsiguda, Hyderabad - 500007 \\ ${ }^{3}$ Current address: Dr. Reddy’s Laboratories, Bachupally, Hyderabad 500090, India
}

Running title- Biophysical investigation of Hsp70-IAPP interactions

"Towhom correspondence should be addressed: Kanchan Garai, Email: kanchan@ tifrh.res.in

Keywords: Hsp70, amyloid oligomer, single molecule detection, burst analysis, FRET, multiangle light scattering, holdase, chaperone amyloid interaction, Field Flow Fractionation 


\begin{abstract}
Molecular chaperone Hsp70 plays important roles in the pathology of amyloid diseases by inhibiting aberrant aggregation of proteins. However, mechanism of the interactions of Hsp70 with the amyloidogenic intrinsically disordered proteins (IDPs) is not clear. Here, we use Hsp70 from different organisms to show that it inhibits aggregation of Islet amyloid polypeptide (IAPP) at substoichiometric concentrations even in absence of ATP. The effect is found to be the strongest if Hsp70 is added in the beginning of aggregation but progressively less if added later, indicating role of Hsp70 in preventing primary nucleation possibly via interactions with the prefibrillar oligomers of IAPP. Fluorescence Correlation Spectroscopy (FCS) measurements of the solutions containing fluorescently labelled Hsp70 and IAPP exhibit fluorescence bursts suggesting formation of heterogeneous complexes of oligomeric IAPP binding to multiple molecules of Hsp70. Size exclusion chromatography and field flow fractionation are then used to fractionate the smaller complexes. Multiangle light scattering and FCS measurements suggest that these complexes comprise of monomers of Hsp70 and small oligomers of IAPP. However, concentration of the complexes is measured to be a few nanomolar amidst several $\mu$ molar of free Hsp70 and IAPP. Hence, our results indicate that Hsp70 interacts poorly with the monomers but strongly with oligomers of IAPP. This is likely a common feature of the interactions between the chaperones and the amyloidogenic IDPs. While strong interactions with the oligomers prevent aberrant aggregation, poor interaction with the monomers avert interference with the functions of the IDPs.
\end{abstract}




\section{Introduction}

Pathology of type 2 diabetes mellitus (T2DM) is characterized by dysfunction of pancreatic $\beta$-cells, progressive decline in insulin secretion and loss of the $\beta$-cell mass. Aetiology of $\beta$-cell damage is multifactorial (1), however, growing evidences suggest that amyloid aggregation of the Islet amyloid polypeptide (IAPP) is a major cause of death of the $\beta$-cells, particularly at the late stage of T2DM $(2,3)$. IAPP, a 37-residue peptide hormone, is co-expressed and co-secreted with insulin from the pancreatic $\beta$-cells. The triggers for aggregation of IAPP in the pancreas is not clear but several different mechanisms such as defects in IAPP biosynthesis, folding, trafficking, and/or processing in response to insulin resistance and inflammation have been proposed to be responsible (4,5). In vitro, IAPP aggregates into soluble oligomers and insoluble fibrils at micromolar concentrations (6). Aggregates of IAPP, particularly the soluble oligomers are considered to be the major toxic species affecting cellular integrity (7-9).

Heat shock protein Hsp70 is a central component of the cellular chaperone system playing essential roles in protein homeostasis under normal and stress conditions by preventing protein misfolding and aggregation $(10,11)$, and protecting the cells from the cytotoxicity caused by amyloid aggregation (12). Expression of Hsp70 is found to be lower in T2DM in the insulin sensitive tissues $(13,14)$. Furthermore, overexpression of Hsp70 in obese mice can delay or prevent the symptoms of diabetes such as inflammation and insulin resistance (15). Chaperone complementation studies either by overexpression or by exogenous addition are shown to minimise the cytotoxic effects of the amyloids in model organisms of T2DM and other amyloid diseases $(16,17)$. Furthermore, Hsp70 is found co-deposited with IAPP in the islet amyloids in T2DM (18). Therefore, elevation of the levels of Hsp70 and also improving its specificity for IAPP are being proposed as therapeutic strategies in $\operatorname{T2DM}(16,19,20)$.

In vitro, Hsp70 along with the cochaperones Hsp40 and the nucleotide exchange factor (NEF) have been reported to inhibit aggregation of amyloid- $\beta, \alpha$-synuclein, polyglutamine polypeptides, tau protein and IAPP (21-25). Furthermore, the Hsp70 machinery can also cause disaggregation of the soluble oligomers and/or the insoluble fibrils in presence of ATP (26). The inhibition of aggregation and disaggregation of preformed aggregates requires only substoichiometric concentrations of Hsp70. Hence, Hsp70 is hypothesized to act in an ATP dependent catalytic cycle, which involves binding to the exposed hydrophobic patches of the 'misfolded' or the 'aggregation competent' conformers of the globular proteins, refolding it, and then releasing it in the 'non-amyloidogenic' monomeric forms (27). Since intrinsically disordered proteins (IDP) do not possess a stable structure it is probably not meaningful to think about a 'misfolded' IDP. Hence, it is unclear how its interaction with the chaperones is regulated. Recent studies have shown ATP independent inhibition of amyloid aggregation by Hsp70 and several other chaperone proteins $(28,29)$. For example, Loomes et al have reported that Hsp70 delays aggregation of IAPP at substoichiometric (1:200) concentrations even in absence of ATP (25). Therefore, inhibitory action of Hsp70 does not necessarily require the catalytic 
activity. However, substoichiometric nature of the interactions indicates that Hsp70 must interact with one or more on-pathway intermediates of IAPP. However, biophysical characterization of these intermediates has been challenging due to its transient and heterogeneous nature (30). Furthermore, Hsp70 is also known to exist as monomers, dimers and higher oligomers (31). Hence, little is known about the properties of the Hsp70/IAPP complexes. We think that investigations using single molecule techniques can help to overcome the limitations posed by the heterogeneity and the transient nature of the interacting species.

Here we have used three different Hsp70 chaperone proteins, such as, E. coli. DnaK (UniProt ID-P0A6Y8), Mycobacterium tuberculosis Hsp70 (MTB-Hsp70, UniProt ID-P9WMJ9) and human Hsc70 (UniProt ID-P11142) and examined its inhibitory effects on different phases, viz, the lag and the growth phase of the aggregation of human IAPP. Then we have measured the size of the Hsp70/IAPP complexes using Multiangle light scattering (MALS) and Fluorescence Correlation Spectroscopy (FCS). Size Exclusion Chromatography (SEC) and Field Flow Fractionation (FFF) are employed to ameliorate the heterogeneity of the complexes. Our results indicate that Hsp70 binds to the prenucleation oligomers to inhibit the growth of the amyloids of IAPP.

\section{Results}

Inhibition of amyloid aggregation of IAPP by Hsp70 proteins. First, we examined whether Hsp70 inhibits aggregation of IAPP in absence of ATP. Figure 1A-C show that time course of aggregation of IAPP $(20 \mu \mathrm{M})$ is characterized by a lag $(\mathrm{t}=0-4 \mathrm{~h})$, a growth $(\mathrm{t}=4-12 \mathrm{~h})$ and a saturation phase $(\mathrm{t}>15$ h) consistent with the nucleation dependent aggregation of amyloid proteins (32). Addition of substoichiometric concentrations $(0,0.125,0.25$ and $0.5 \mu \mathrm{M})$ of DnaK, MTB-Hsp70 or Hsc70 delays the kinetics of aggregation dramatically. While all the three chaperones inhibit amyloid aggregation, there are some differences. For example, the inhibitory effects appear to be the strongest in presence of DnaK and weakest in presence of Hsc70. The sequence identity between the Hsp70 proteins used here is about 50\% (33). Therefore, despite large differences in the primary sequences, Hsp70 across different species share common mechanisms in protection against amyloid aggregation. This is consistent with the highly conserved nature of the overall structural organization of the variants of Hsp70 (33).

Figure 1D examines whether the inhibitory effects of Hsp70 could be enhanced in presence of ATP. It may be seen that the inhibitory effects of DnaK on the kinetics of aggregation of IAPP in presence and absence of ATP are quite similar. This is not surprising because inherent ATPase activity of Hsp70 is known to be poor (11).

Some literature reports suggest that Hsp70 prevents amyloid formation, but it promotes amorphous aggregation (28). We have performed Atomic Force Microscopy (AFM) measurements on the aggregates of IAPP prepared upon incubation with or without DnaK. Clearly, large quantities of amyloid fibrils are observed in the sample containing IAPP alone (Figure 1E). However, aggregation 
observed in presence of DnaK is minimal (Figure 1F). Thus, Hsp70 appears to inhibit both fibrillar and non-fibrillar growth of IAPP.

Inhibition of the aggregation of IAPP in the absence of ATP indicates that inhibitory action of Hsp70 is not mediated by the catalytic cycle of 'foldase' activity rather it is mediated possibly by the 'holdase' activity (25). However, interactions of Hsp70 with monomeric IAPP can't explain the strong effects observed at sub-stoichiometric concentrations (21). Therefore, Hsp70 must interact with one or more on-pathway intermediates of IAPP. The intermediates could be the pre-nucleation oligomers, post-nucleation protofilaments or even 'misfolded' monomers.

Inhibitory effect of Hsp70 is the strongest at the prenucleation phase. We then examined whether the inhibitory role of Hsp70 is mediated via its interactions with the prenucleation oligomers or with the post-nucleation fibrillar species. Figure $2 \mathrm{~A}$ shows the time course of aggregation of a $20 \mu \mathrm{M}$ IAPP solution. DnaK $(1 \mu \mathrm{M})$ is added into this solution in the beginning (i.e., at $\mathrm{t}=0$ ), in the lag phase (at $\mathrm{t}$ $=3 \mathrm{~h}$ ) and in the growth phase (at $\mathrm{t}=5 \mathrm{~h}$ ). The inhibitory effect of DnaK is clearly the highest if it is added at $\mathrm{t}=0$. The effect is progressively less if added later. Addition of the chaperone in the "postnucleation' phase slows down the growth of the fibrils but it doesn't stop it. Figure 2B shows that similar observations are made using $\mathrm{Hsc70}$ as well. Taken together these results indicate that primary mechanism of the inhibitory action of Hsp70 possibly involves prevention of the primary nucleation of IAPP than prevention of the growth of the fibrils.

Binding of DnaK with the prefibrillar species of IAPP monitored using Intermolecular Forster Resonance Energy Transfer (FRET). To monitor intermolecular FRET, we have used EDANS-DnaK and TMR-IAPP. Figure 3A shows that the kinetics of intermolecular FRET exhibit two distinct phases, a fast phase ( $\mathrm{t}<$ mixing time of the samples, i.e., $\mathrm{t}<5$ seconds) and a slow phase $(\mathrm{t}>1 \mathrm{~h})$. The fast phase most likely indicates binding with the preformed oligomers and/or with the monomers of IAPP. The extremely slow kinetics of the second phase is consistent with binding of DnaK with the oligomers of IAPP that form slowly with time. Therefore, formation of the oligomers is likely the rate limiting in this phase. This is further supported by the observation that the intermolecular FRET reaches completion faster if the concentration of EDANS-DnaK used is lesser, as it can be 'consumed' by relatively lower concentration of the oligomers.

To examine the time evolution of the aggregation status of TMR-IAPP, we monitored the fluorescence of TMR under the same experimental conditions following the assay developed by Garai and Frieden (32). The kinetics of fluorescence of TMR-IAPP exhibit in three distinct phases (Figure 3B). These different phases are interpreted as the oligomerization phase $(\mathrm{t}<2 \mathrm{~h})$, the 'lag' phase $(\mathrm{t}$ $10 \mathrm{~h}$ ) and the growth phase (32). In presence of DnaK, the 'lag' phase is extended beyond $30 \mathrm{~h}$. The data presented in Figure 3A indicate that the intermolecular FRET reaches saturation in about $10 \mathrm{~h}$. Thus, intermolecular FRET occurs primarily during the oligomerization and the 'lag' phase of aggregation. Therefore, binding of DnaK must involve primarily the prefibrillar species of IAPP. 
Ensemble and single molecule measurements of the binding constant of DnaK/IAPP interactions reflect heterogeneity of the complexes. To determine the binding constant of the interactions, we measured fluorescence anisotropy of TMR-IAPP in the presence of unlabeled DnaK. Since IAPP is a small peptide, anisotropy of TMR-IAPP $(\mathrm{MW}=4.4 \mathrm{kDa})$ is expected to increase considerably upon binding to DnaK (MW = $69 \mathrm{kDa})$. Figure 4A shows that anisotropy of $400 \mathrm{nM}$ TMR-IAPP increases in presence of 0 to $5 \mu \mathrm{M}$ DnaK in a concentration dependent manner. While the increase in anisotropy of TMR-IAPP indicates binding to DnaK, the increase is quite small $(\approx 20 \%)$. The equilibrium constant $\left(K_{e q}\right)$ obtained from fitting the data using a single site binding model is $2.1 \pm 0.9 \mu \mathrm{M}$, which appear very high considering that even a few nanomolar DnaK is shown to alter aggregation of IAPP strongly (see Figure 1).

To examine the interactions further we use a single molecule technique, viz, Fluorescence Correlation Spectroscopy (FCS) to measure the hydrodynamic radius $\left(\mathrm{R}_{\mathrm{h}}\right)$ of TMR-IAPP in presence of DnaK. Since $\mathrm{R}_{\mathrm{h}}$ is proportional to $\mathrm{MW}^{1 / 3}$, it is expected to increase by $\sim 2.6$-times if IAPP binds to monomeric DnaK. Figure 4B shows that $\mathrm{R}_{\mathrm{h}}$ of TMR-IAPP increases in presence of 0 to $5 \mu \mathrm{M}$ DnaK in a concentration dependent manner. Surprisingly, the shape of the $R_{h}$ versus [DnaK] plot doesn't conform to that is expected from a single site binding model as observed in case of the anisotropy data presented in Figure 4A. Furthermore, the increase of $R_{h}$ even in presence of $5 \mu \mathrm{M}$ DnaK is only about $30 \%$, which is significantly smaller than the expected $160 \%$ increase if all the monomeric IAPP are bound to the DnaK. Therefore, most of the IAPP in the solution must be free. Thus, it is possible that DnaK binds primarily to the oligomers of IAPP. Small increase of anisotropy or $\mathrm{R}_{\mathrm{h}}$ is indicative of the presence of a relatively small population of the oligomers of IAPP. To examine presence of the oligomers, we compared the photon count traces and the autocorrelation traces. Figure 4C and D show typical examples of photon count and autocorrelation traces. Clearly, a significant number of photon count traces exhibit presence of fluorescence bursts indicating presence of oligomers of IAPP. Consequently, large variability is observed among the autocorrelation curves as well. Therefore, FCS data indicate presence of oligomers in the solution. Higher brightness of the oligomeric complexes leads to the heterogeneities in the FCS measurements. We note here that presence of bursts distorts the autocorrelation data (see Figure 4D), hence these data are excluded from the analysis. Thus, nature of the binding curves obtained from the ensemble anisotropy and FCS measurements could differ considerably (see Figure 4A and B).

It may be argued that the above-mentioned heterogeneity is a characteristic of TMR-IAPP. To verify this, we performed FCS measurements of TMR-DnaK in presence of unlabeled IAPP. Many fluorescence bursts are observed in the photon count traces of TMR-DnaK in presence (Figure 5C) but not in absence of IAPP (Figure 5A). The autocorrelation traces obtained in presence of IAPP are also highly heterogeneous as may be expected (Figure 5D). The observed fluorescence bursts are indicative of clustering the TMR-DnaK molecules possibly on the oligomers of IAPP. Quantification of the oligomers in the midst of a vast majority of the free monomers is difficult (34). However, 
comparison of the photon counting histogram $(\mathrm{PCH})$ of TMR-DnaK in presence and absence of IAPP shows that about $8 \%$ of the total photons are associated with the photon bursts (Figure 5E). Hence, about $8 \%$ of the $100 \mathrm{nM}$ TMR-DnaK are associated with the complexes giving rise to the bursts. Then we used a photon count threshold of mean $+2 \times$ standard deviation (SD) to count the number of the bursts per minute. We observed about 16 photon bursts per minute. Furthermore, the probability of detecting a photon burst in any time bin (= no. of the bins associated with the bursts/total no. of bins) is found to be about 0.01. In our FCS setup, the average number of molecules $(<\mathrm{N}\rangle)$ observed in the confocal volume from $1 \mathrm{nM}$ solution is about 1.1 (35). Hence, the number concentration of the oligomeric complexes must be in the low picomolar range (36). The size of the photon bursts exhibits a broad distribution (Figure 5F). The peak of the distribution corresponds to about $130 \mathrm{kHz}$. Counts per molecule (CPM) of the monomeric TMR-DnaK obtained from the FCS measurement of the purified TMR-DnaK solution is about $8 \mathrm{kHz}$ (procedure to determine CPM is described in (37). Hence, the average brightness of the complexes are about 16-times higher than monomeric TMRDnaK. Therefore, oligomers of IAPP interact with high affinity with multiple molecules of DnaK. We note here that the approach outlined above is suitable only for the large bursts, as the small complexes would get masked in the shot noise of the photon counts. Hence, this approach is not suitable for characterization of the small complexes.

Characterization of the complexes of DnaK/IAPP by Multiangle Light Scattering (MALS) and FCS following fractionation by SEC and FFF. The FCS measurements presented above indicate that the major problem in characterization of the DnaK/IAPP interaction is heterogeneity of the complexes. Therefore, we use SEC to resolve the various species in the heterogeneous mixture of free IAPP, free DnaK and the IAPP/DnaK complexes. The SEC is connected to a MALS instrument for determination of the absolute molecular weight and the size of the DnaK/IAPP complexes. Figure 6A shows the time course of the concentration of the eluting proteins measured by refractive index (RI) of the solution. It may be seen that the RI (i.e., the concentration) profile shows two closely spaced peaks at 43 and 45 mins. The same figure shows the time course of the molecular weight (MW) obtained from analysis of the MALS data. It may be seen that the MW of the particles eluted corresponding to the peak of the RI is measured to be $70-80 \mathrm{kDa}$ indicating that DnaK exists predominantly as monomers. To verify if the two peaks correspond to complexes of DnaK with different oligomers of IAPP we compared the data obtained from a sample containing DnaK alone. However, the profiles of the RI and the MW of the samples containing DnaK/IAPP and DnaK alone appear to be similar. Then, we performed mass spectrometry measurements on the samples corresponding to the peaks observed in RI to obtain an estimate of the relative concentrations of Dnak and IAPP in the DnaK/IAPP sample. Supplementary figure 1 shows that the peaks contain DnaK. However, no IAPP is detectable in these fractions. This indicates that the population of the DnaK/IAPP complexes must be small. The peaks observed in the RI and the MALS must originate from the primarily free DnaK. The two peaks in RI might indicate slow monomer-dimer equilibrium of DnaK (31). To characterize the DnaK/IAPP 
complexes specifically we then used FCS. In these experiments we used TMR-IAPP instead of IAPP. Since DnaK used here is not fluorescently labelled the unbound DnaK doesn't contribute to the signal in FCS. First, SEC is used to fractionate the DnaK/TMR-IAPP complexes from the population of the free TMR-IAPP. Figure 6B shows that the $\mathrm{R}_{\mathrm{h}}$ of TMR-IAPP/DnaK complex measured using FCS is $\sim 3.3 \mathrm{~nm}$, which is almost same as that of the monomeric DnaK but quite larger than that of the free IAPP $\left(\mathrm{R}_{\mathrm{h}} \sim 1.2 \mathrm{~nm}\right)$. Therefore, FCS measurements indicate binding of monomeric DnaK to monomers or small oligomers of TMR-IAPP. Since $R_{h}$ is proportional to $(M W)^{1 / 3}$, the FCS measurements do not have the resolution to distinguish if these complexes contain monomers or small oligomers of IAPP. However, the concentration of the TMR-IAPP/DnaK complexes detected in FCS is approximately $45 \mathrm{nM}$ (Supplementary Table S1). We note here the concentrations of DnaK and IAPP used in these experiments are several $\mu \mathrm{M}$. Therefore, concentration of the complexes detected in FCS is very low suggesting that most of the DnaK or the TMR-IAPP in the solution are free.

A well-recognized problem in characterization of non-covalent protein-protein complexes by SEC-MALS is that the complexes may dissociate due to interactions of the proteins with the matrix of the SEC column. To circumvent this problem, we attempt to resolve the various species of the IAPP/DnaK complexes by using Field Flow Fractionation (FFF), which uses flow field to separate the molecules based on hydrodynamic size (38). Figure 6C compares the time courses of the RI and the MW obtained from the mixture of DnaK and IAPP, and the DnaK alone. It may be seen that both the samples show two closely spaced peaks at 12 and 13 mins in RI. The MW of the peaks are found within 60-80 kDa consistent with the SEC-MALS measurements. Once again, we performed FCS measurements on the TMR-IAPP/DnaK samples 'purified' using FFF. Analysis of the FCS data presented in Figure $6 \mathrm{~B}$ show that $\mathrm{R}_{\mathrm{h}}$ of the complexes are almost same as the monomeric DnaK. Once again, the concentration of the complexes is very low ( $15 \mathrm{nM})$ (Supplementary Table S1). Therefore, FFF-MALS data are consistent with the SEC-MALS data. The FCS autocorrelation data and the fits are shown in supplementary figure S2. Taken together, the FCS data indicate that the $\mathrm{R}_{\mathrm{h}}$ of the complexes are same as the complex of monomeric DnaK binding to monomers or small oligomers of IAPP. Since, the binding of DnaK involves a small fraction of the population of IAPP, we think that DnaK binds to the small oligomers of IAPP.

\section{Discussion}

Amyloid aggregation of proteins is involved in the pathology of multiple human diseases such as Alzheimer's, Parkinson's disease and T2DM. Extensive studies over the past three decades indicate that protein monomers first assemble into soluble low molecular weight oligomers, the oligomers then act as sites for nucleation to form small fibrillar aggregates, which grow to larger fibrils. To counteract the numerous pathways that lead to aberrant aggregation of proteins, cells utilise molecular chaperones as a defence mechanism $(10,12,39,40)$. Hsp70 chaperone machinery is the central component of the chaperone systems providing protection against aberrant aggregation of cellular 
proteins under stress conditions. The commonly depicted mechanism of action is that Hsp70 machinery, which involve the cochaperones such as Hsp40 and the nucleotide exchange factors (NEF), bind to the exposed hydrophobic patches of the fully unfolded polypeptide chains or 'misfolded' proteins, and then release it in the correctly 'folded' form using an ATP dependent catalytic cycle (41). In vitro, Hsp70/Hsp40/NEF machinery has been shown to inhibit aggregation and promote disaggregation of the aggregates of amyloid- $\beta, \alpha$-synuclein and polyglutamine polypeptides in presence of ATP $(21,25)$. In vivo, over expression or exogeneous addition of Hsp70 has been shown to inhibit amyloid aggregation and rescue cytotoxicity of the amyloids in cell cultures and model organisms $(16,20,42)$.

While the 'foldase' activity of Hsp70 is mediated by the ATP dependent catalytic cycle several recent in vitro studies have shown that Hsp70 can inhibit amyloid aggregation also in absence of ATP suggesting that the catalytic cycle is not required for the inhibitory action of Hsp70 $(12,25)$. Therefore, Hsp70 can act as a 'holdase' in absence of the cochaperones and ATP. This is not surprising as binding of Hsp70 to the polypeptide might delay its aggregation by blocking the sites of growth. However, the extent of the delay would depend on the molecular mechanism of the interactions. For example, if Hsp70 binds predominantly to the monomers then in absence of ATP driven catalytic activity stoichiometric amount of Hsp70 is required to observe significant effects. Our results presented in Figure 1-3 show that Hsp70 can exert strong effects even at substoichiometric concentrations indicating that it must interact with the intermediates/oligomers rather than the monomers of IAPP. This is further supported by the intermolecular FRET measurements which show that DnaK interacts with species of IAPP that form in a time dependent manner (see Figure 3).

However, very little is known so far about the biophysical properties of the DnaK/IAPP complexes. The fluorescence bursts of the TMR-DnaK formed in presence of IAPP suggest that the oligomers of IAPP bind to multiple molecules of DnaK. We find that the concentration of the bursts, i.e., concentration of the complexes is in the picomolar range. Size of the oligomeric complexes could not be determined, however, average brightness of the bursts suggests about 10-100 molecules of DnaK per oligomers. An inherent limitation of the burst analysis in presence of a high background fluorescence, which arises from the large population of the free TMR-DnaK is that the small oligomers cannot be detected or characterized. Therefore, SEC and FFF are used to separate the large oligomeric complexes. We find that the large oligomeric complexes are eliminated by SEC or FFF. While MALS cannot distinguish DnaK/IAPP complexes from free DnaK, FCS can provide information on the size and concentration of the complexes. FCS and MALS measurements on the major fractions indicate that the DnaK/IAPP complexes are small $\sim 90 \mathrm{kDa}$. Thus, these complexes most likely contain one molecule of DnaK and one or a few molecules of IAPP. However, the concentration of these complexes is very low $\sim 15-45 \mathrm{nM}$. Since the concentration of the complexes are very low we think that the interaction involves a rare species, i.e., the oligomers of IAPP. Therefore, majority of the DnaK and the IAPP in the mixture must be free. Therefore, our 
experiments suggest that binding of DnaK with the monomers of IAPP is poor, while affinity for binding to the oligomers is very high.

Our results presented in Figure 2 indicate that the effects of DnaK when added at $t=0$ is the strongest. Therefore, DnaK appears to be more effective on the prefibrillar oligomers than the fibrillar forms. Hence, we hypothesize that major role of DnaK is to interact with the oligomers to prevent nucleation rather than preventing growth of the preformed fibrils. The mechanism of the interaction of Hsp70 with substrate proteins has been a matter of intense studies (41,43-46). Hsp70 is known to be highly promiscuous due to its interactions with multitude of substrates with diverse polypeptide sequences (33). However, most of our knowledge about how Hsp70 binds to its substrates is based on the studies using model peptides from experiments using peptide libraries and phage display $(47,48)$. The octapeptide substrate for Hsp70 generally contains a six-residue hydrophobic middle patch flanked by positively charged residues at both ends. Many polypeptide sequences contain such patches. Therefore, Hsp70 binds to fully unfolded polypeptides or the exposed hydrophobic patches in the globular proteins. For example, Hsp70 machinery can refold denatured luciferase in vitro (49). Therefore, binding of Hsp70 with globular proteins is regulated by misfolding or unfolding of the protein. However, little is known about how Hsp70 binds to intrinsically disordered proteins (IDP) since the putative binding motifs in IDPs are possibly exposed almost all the time. Therefore, how is the interaction with the IDPs regulated? Molecular chaperone binding site prediction program LIMBO (50) predicts residues 13-19 heptapeptide (ANFLVHS) of IAPP as a probable binding site for DnaK. Therefore, Hsp70 may bind to the patch containing ANFLVHS in the monomeric IAPP. However, our experiments suggest that DnaK binds strongly to the oligomers but weakly to the monomers. Therefore, we think that the high affinity of the interaction with the oligomers arises from multivalent binding. The multivalent binding might involve oligomeric forms of IAPP and the multiple binding sites on Hsp70, similar to what has been proposed earlier for the interaction between apolipoprotein $\mathrm{E}$ and the oligomers of amyloid- $\beta$ (51). While the polypeptide substrates are believed to bind to the canonical substrate binding cleft in the substrate binding domain, the surface of the Hsp70 proteins are capable of interacting with multitude of cochaperone proteins (33). In fact, diversity of the functions of Hsp70 are hypothesised to originate from its ability to interact with diverse array of cochaperones (52). Furthermore, a second binding site that is important for the activity of the Hsp70 has been reported recently (53). The multivalent binding might involve this second binding site and/or other protein-protein interaction sites on the surface of Hsp70. Unlike folded proteins since the IDPs do not possess defined structure, high affinity binding of Hsp70 to the oligomeric but not the monomeric forms of IAPP possibly is required to regulate the interaction ensuring that the cellular functions of the monomeric IAPP are not affected. Furthermore, FCS measurements reported in Figure 5 suggest that oligomers of IAPP bind to multiple molecules of Hsp70. Recruitment of multiple Hsp70 molecules to the protein rhodanase has been hypothesized to promote unfolding of the 
protein via an 'entropic pulling' action (54). Similar mechanism of action may be involved in the disassembly of the oligomers or the fibrils of the amyloid proteins by the Hsp70 machinery (26).

Thus, our data supports that Hsp70 alone can inhibit aggregation of IAPP by the 'holding' model $(21,25)$. Binding to the oligomers of IAPP is probably the first step in the catalytic cycle of the chaperone machinery $(21,25)$. Alternatively, Hsp70 can direct the bound complexes to the degradation pathways (55). Several other heat shock proteins such as Hsp90 and Hsp60 also exhibit similar protection against amyloid aggregation $(21,56)$. We find that the Hsp70 binds weakly with the monomers but strongly with affinity in the picomolar range with the oligomeric forms of IAPP. This might be a common feature, which is important for the regulation of the interactions between the constitutively expressed molecular chaperones and the functionally important amyloidogenic IDPs.

\section{Experimental Procedures}

Preparation of IAPP and Hsp70 chaperones: All the chemicals unless mentioned otherwise were procured from Sigma (USA). Tetramethylrhodamine (TMR)-maleimide and EDANS-maleimide were purchased from Thermo Fischer Scientific (USA).

Chemical synthesis of IAPP: IAPP was chemically synthesised at $0.1 \mathrm{mmol}$ scale using an automated peptide synthesiser (Aapptec) following a protocol described earlier by Marek et al (57). Crude IAPP was dissolved in $30 \mathrm{mM}$ Acetic acid. The disulfide bond between the cysteines at positions 2 and 7 was induced using hydrogen peroxide. The peptide was stored in the form of lyophilized powder at $-20{ }^{\circ} \mathrm{C}$. The lyophilized power was dissolved in $10 \mathrm{mM} \mathrm{pH} 7.4$ phosphate buffer containing 4M Guanidine hydrochloride $(\mathrm{GdnCl})$. This solution was purified by size exclusion chromatography using a superdex peptide column (10/300, GE Healthcare, Germany) in $20 \mathrm{mM}$ phosphate $\mathrm{pH} 7.4$ buffer containing $4 \mathrm{M} \mathrm{GdnCl}$. Only the monomeric fraction was collected. Finally, a PD10 column (GE Healthcare, Germany) was used to remove the $\mathrm{GdnCl}$. The stock solution was aliquoted into $100 \mu \mathrm{l}$ vials, flash frozen and stored at $-80^{\circ} \mathrm{C}$. The mass and purity of the peptide was verified by mass spectrometry (see supplementary figure 3 ).

Labeling of IAPP with TMR. Tetramethylrhodamine (TMR) was covalently linked to the Nterminus of IAPP before cleaving the peptide from the resin. Briefly, the N-terminus of IAPP was deprotected using $20 \%$ piperidine followed by extensive wash with dimethylformamide (DMF). 5,6carboxy TMR was then added in 2-fold molar excess and the reaction mixture was kept overnight at RT with stirring. The resin was washed with DMF followed by dichloromethane and methanol to remove free TMR. The peptide was lyophilised and then cleaved from the resin and purified using the procedure mentioned above.

Expression and purification of $E$. coli DnaK, MTB-Hsp70 and Human Hsc70. Plasmids for $E$. coli DnaK, Mycobacterium tuberculosis Hsp70 and human $H s c 70$ are generous gifts from Dr. Shekhar Mande (National Centre for Cell Science, Pune, India), Prof. Lila Gierasch (University of 
Massachusetts, USA) and Prof. Lewis Kay (University of Toronto, Canada) respectively. Expression and purification of the proteins were performed using the published protocols $(46,58,59)$. BL21 (DE3) E. coli cells were transformed with the plasmids described above. Cells were grown in Luria Broth (LB) supplemented with $100 \mu \mathrm{g} / \mathrm{ml}$ ampicillin at $37{ }^{\circ} \mathrm{C}$ with continuous shaking of $200 \mathrm{rpm}$. Expression of the proteins was induced with $1 \mathrm{mM}$ IPTG at 0.6 OD of cell density. The cells were harvested by centrifugation after 4 hours of induction. DnaK expressing cells were resuspended in 50 mM Tris pH8.0 and $1 \mathrm{mM}$ EDTA buffer (Buffer A) and lysed using sonication (Branson, USA). The cell lysate was centrifuged at $18000 \mathrm{rpm}$ for 40 minutes using a floor centrifuge (Beckman, USA). The supernatant, which contains DnaK protein was purified first with ion exchange chromatography using 0 to $1 \mathrm{M} \mathrm{NaCl}$ in a HiTrap column (HiTrap, GE Healthcare, Germany). Primary fraction of DnaK was eluted in $150 \mathrm{mM} \mathrm{NaCl}$ which was further purified using size exclusion chromatography using a superdex 200 column (GE Healthcare, Germany) in PBS, pH 7.4 buffer containing $1 \mathrm{mM}$ EDTA and $1 \mathrm{mM}$ sodium azide. The fraction containing monomeric DnaK was collected, aliquoted into $50 \mu \mathrm{l}$ vials, flash frozen and stored at $-80{ }^{\circ} \mathrm{C}$.

Hsc70 and MTB-Hsp70 were purified first by Ni-NTA column chromatography (His-trap, GE Healthcare). The column was washed with $50 \mathrm{mM}$ Tris $\mathrm{pH} 8,500 \mathrm{mM} \mathrm{NaCl}$ and $20 \mathrm{mM}$ imidazole. The proteins were eluted using $400 \mathrm{mM}$ imidazole. N-terminal His-tag of Hsc70 was removed using TEV protease (1:50 ratio of TEV:Hsc70). The proteins were finally purified using size exclusion chromatography using a Superdex 200 column (10/300 GL, GE Healthcare, Germany) equilibrated with PBS buffer, pH 7.4 containing $1 \mathrm{mM}$ EDTA and $1 \mathrm{mM}$ sodium azide. The pure monomeric fractions of MTB-Hsp70 and Hsc70 were aliquoted in $50 \mu \mathrm{l}$ vials, flash frozen and stored at $-80{ }^{\circ} \mathrm{C}$. The purity of the proteins was examined by SDS-PAGE and mass spectrometry (see supplementary figure 4).

Measurement of kinetics of aggregation of IAPP. A $100 \mu \mathrm{M}$ stock solution of IAPP was diluted to $20 \mu \mathrm{M}$ into phosphate buffer saline (PBS) at $\mathrm{pH} 7.4$ containing $4 \mu \mathrm{M}$ Thioflavin $\mathrm{T}$ (ThT) into a round bottom glass test tube $(\mathrm{OD}=10 \mathrm{~mm})$ placed inside a temperature-controlled cell holder in the spectrofluorometer (PTI, USA). The solution was stirred continuously using a teflon coated microstirrer $(3 \mathrm{~mm} \times 6 \mathrm{~mm})$ bead at approximately $300 \mathrm{rpm}$ at $37{ }^{\circ} \mathrm{C}$. Fluorescence of ThT was monitored continuously using excitation $\left(\lambda_{\text {ex }}\right)$ at $438 \mathrm{~nm}$ and the emission $\left(\lambda_{\text {em }}\right)$ at $480 \mathrm{~nm}$. Substoichiometric concentrations $(125,250$ and $500 \mathrm{nM})$ of DnaK or MTB-Hsp70 or Hsc70 were added to $20 \mu \mathrm{M}$ IAPP to examine the effects of the chaperone proteins on the kinetics of aggregation of IAPP. To characterize the effects of Hsp70 on various stages of aggregation, $1 \mu \mathrm{M}$ DnaK (or Hsc70) was added to $20 \mu \mathrm{M}$ IAPP in the initial $(\mathrm{t}=0 \mathrm{~h}), \operatorname{lag}(\mathrm{t}=3 \mathrm{~h})$ and in the growth phase $(\mathrm{t}=5 \mathrm{~h})$ of aggregation of IAPP. Aggregation of TMR-IAPP $(2 \mu \mathrm{M})$ was performed at pH 7.4 in PBS buffer at $37{ }^{\circ} \mathrm{C}$ with continuous stirring at $300 \mathrm{rpm}$. The fluorescence of TMR was monitored continuously at $\lambda_{\mathrm{em}}=600 \mathrm{~nm}$ with $\lambda_{\mathrm{ex}}=520 \mathrm{~nm}(32)$. 
Atomic force microscopy (AFM) imaging. A $10 \mu \mathrm{l}$ IAPP/DnaK $(20 \mu \mathrm{M} / 0.5 \mu \mathrm{M})$ solution was collected from the end of the experiment involving kinetics of aggregation described above. The solution was adsorbed onto a freshly cleaved mica sheet for about 2 minutes. The surface of the mica was then washed gently with Milli-Q water to remove the salt and the loosely bound aggregates. The sheet was then dried in an enclosed chamber for overnight at room temperature. The sample was imaged using an AFM (AFM workshop, CA, USA) in the tapping mode. The resonance frequencies of the cantilever used is within $140-210 \mathrm{kHz}$ and the nominal radius of curvature of the tip used in the measurement is $<20 \mathrm{~nm}$. The scanning area is $10 \times 10 \mu \mathrm{m}$. The scan rate used is $0.5 \mathrm{~Hz}$.

Kinetics of interaction between DnaK and IAPP by FRET. DnaK was fluorescently labelled at the native cysteine residue at position 6 with EDANS-maleimide using the standard maleimide labeling protocol. EDANS-DnaK was purified using a superdex 200 column (10/300 GL, GE Healthcare, Germany) in PBS buffer at pH 7.4. Different concentrations of EDANS-DnaK (150, 300, $600 \mathrm{nM})$ were added to TMR-IAPP $(2 \mu \mathrm{M})$ in PBS buffer, $\mathrm{pH}$ 7.4. The FRET is monitored continuously using the donor (EDANS) fluorescence using $\lambda_{\mathrm{ex}}=350 \mathrm{~nm}$ and $\lambda_{\mathrm{em}}=480 \mathrm{~nm}$ in the spectrofluorometer (PTI, USA). FRET efficiency was calculated using, FRET efficiency $E=1-F_{D A} / F_{D}$, where $F_{D A}$ is donor emission obtained in presence of the acceptor and $F_{D}$ is donor fluorescence in the absence of acceptor (51). $F_{D}$ was obtained from the fluorescence of EDANS-DnaK monitored in presence of 2 $\mu \mathrm{M}$ unlabeled-IAPP.

Measurement of DnaK and IAPP interactions using fluorescence anisotropy. Purified $400 \mathrm{nM}$ TMR-IAPP was incubated with different concentrations of DnaK (0 to $5 \mu \mathrm{M}$ ) in PBS buffer ( $\mathrm{pH}$ 7.4) containing $1 \mathrm{mM}$ EDTA and $1 \mathrm{mM}$ sodium azide. The instrument ' $\mathrm{g}$ ' factor is calculated using free TMR-IAPP. Fluorescence anisotropy of TMR-IAPP was measured in presence of different concentrations of DnaK. The data were fit using a single site binding model using the following equation.

$Y=\frac{B_{\max } X}{k_{d}+X}$

Here, $Y$ is the anisotropy, $X$ is the concentration of DnaK and $k_{d}$ is the equilibrium binding constant.

Fluorescence correlation spectroscopy (FCS) measurements. The FCS experiments were performed using a homebuilt setup described elsewhere (35). We used two excitation lasers, a solidstate laser with $\lambda=488 \mathrm{~nm}$ (Melles Griot, USA) and a He-Ne laser with $\lambda=543 \mathrm{~nm}$ (Newport, USA). The experiments were performed with TMR-IAPP or TMR-DnaK. Hence, only the He-Ne laser was used. For measurement of binding of TMR-IAPP and DnaK, TMR-IAPP (400 nM) solution was incubated with 0 to $5 \mu \mathrm{M}$ DnaK in PBS buffer, $\mathrm{pH} 7.4$ in presence of $1 \mathrm{mM}$ EDTA and $1 \mathrm{mM} \mathrm{NaN}_{3}$ at RT. The autocorrelation data was analysed using a single component diffusion model. Presence of fluorescence bursts distort the autocorrelation data. Hence, these are removed from the analysis. The diffusion time obtained was used to calculate the hydrodynamic radii $\left(\mathrm{R}_{\mathrm{h}}\right)(35)$. Calibration of the FCS observation volume was performed using free rhodamine B (37). 
Burst analysis. Photon count traces obtained from the FCS measurements of $100 \mathrm{nM}$ TMR-DnaK in presence of $20 \mu \mathrm{M}$ IAPP were recorded with a bin time of $100 \mu \mathrm{s}$. First, the photon counting histogram $(\mathrm{PCH})$ is calculated. To obtain an estimate of the mean and the width (SD) of the $\mathrm{PCH}$, the data are fit using a gaussian function. We note here that the appropriate function to fit the PCH data is quite complex. Since our objective is to estimate the mean and the SD only to decide a threshold for detection of the bursts, we have used fitting of the PCH simply with a Gaussian function. Analysis of the fluorescence bursts were performed using approaches similar to those described in (60). The threshold is set at mean $+2 \times \mathrm{SD}$. The number of bursts, total photon counts $(n)$ per burst and the temporal size $(\tau)$ of the bursts are calculated from the raw photon count data using the free available module 'peakutils' in Python. The brightness of each burst is calculated as, $\epsilon=n / \tau$.

FFF-MALS and SEC-MALS measurements. The FFF and the MALS instruments are from Postnova Analytics (Germany). The FFF was used to separate the DnaK/IAPP complexes from the free IAPP. The MALS is connected in-line with the FFF for the measurements of the molecular weight (MW) and radius of gyration $\left(\mathrm{R}_{\mathrm{g}}\right)$ of the proteins. IAPP $(20 \mu \mathrm{M})$ was incubated with $10 \mu \mathrm{M}$ DnaK in PBS pH 7.4 buffer at RT. A $100 \mu$ aliquot of this mixture was injected in the FFF system. We have used a $5 \mathrm{kDa}$ cellulose membrane, $0.20 \mathrm{ml} / \mathrm{min}$ focus flow for 6 minutes, $0.25 \mathrm{ml} / \mathrm{min}$ forward flow and $3 \mathrm{ml} / \mathrm{min}$ cross flow in the FFF. The $\mathrm{dn} / \mathrm{dc}$ measurements were performed using a refractive index (RI) detector (Postnova Analytics). The SEC-MALS measurements were performed in the same setup. In this case instead of using the FFF membrane assembly a Superdex 200 column was used for fractionation of the DnaK/IAPP complexes. The flow rate used in SEC is $0.4 \mathrm{ml} / \mathrm{min}$.

\section{Acknowledgements}

We acknowledge funding from Department of Atomic Energy, India. This work is partly supported by early career grant received by KG from Science and Engineering Research Board, India.

\section{Conflict of Interest}

The authors declare no conflict of interest.

\section{Author contributions}

KG conceptualized research. NC, BS, DS, SC and MM performed research. KG and BS performed analysis. KG and $\mathrm{NC}$ wrote the paper. 
Figure Legends

Figure 1: Effect of Hsp70 on aggregation of IAPP. A-C) Kinetics of aggregation of $20 \mu$ IAPP in presence of substoichiometric concentrations of E.coli DnaK (A), MTB-Hsp70 (B) and human Hsc70 (C). Ratios indicated in the figures are molar ratios. D) Effect of DnaK $(0.5 \mu \mathrm{M})$ on the kinetics of aggregation of $20 \mu \mathrm{M}$ IAPP in absence or presence of $1 \mathrm{mM}$ ATP. Aggregation is monitored using fluorescence of Thioflavin T. E-F) AFM images of the $20 \mu \mathrm{M}$ IAPP incubated for 48 hours in absence or presence of $0.5 \mu \mathrm{M}$ DnaK. All experiments are performed in PBS buffer containing $1 \mathrm{mM}$ EDTA and $1 \mathrm{mM}$ sodium azide at $\mathrm{pH} 7.4$ and incubated at $37{ }^{\circ} \mathrm{C}$ with continuous stirring.

Figure 2: Effect of Hsp70 on different stages of IAPP aggregation. $1 \mu \mathrm{M}$ DnaK (A) or Hsc70 (B) is added to a $20 \mu \mathrm{M}$ IAPP solution at different stages of aggregation, viz, in the beginning ( $\mathrm{t}=0$ ), 'lag' phase $(\mathrm{t}=3 \mathrm{~h})$ and in the growth phase $(\mathrm{t}=5 \mathrm{~h})$. Clearly, the effects of Hsp70 is highest if added early. Solutions are prepared in PBS buffer containing $1 \mathrm{mM}$ EDTA and $1 \mathrm{mM}$ sodium azide at $\mathrm{pH} 7.4$ and incubated at $37{ }^{\circ} \mathrm{C}$ with continuous stirring.

Figure 3: Kinetics of interaction between EDANS-DnaK and TMR-IAPP. A) Kinetics of intermolecular FRET between EDANS-DnaK (150 to $600 \mathrm{nM}$ ) and TMR-IAPP ( $2 \mu \mathrm{M})$ monitored using donor (EDANS) fluorescence $\left(\lambda_{\mathrm{ex}}=350 \mathrm{~nm}, \lambda_{\mathrm{em}}=480 \mathrm{~nm}\right)$. B) Kinetics of aggregation of TMR-IAPP monitored by fluorescence of TMR in presence and absence of EDANS-DnaK $\left(\lambda_{\text {ex }}=520\right.$ $\left.\mathrm{nm}, \lambda_{\mathrm{em}}=600 \mathrm{~nm}\right)$. Comparison of the time scales between $\mathrm{A}$ and $\mathrm{B}$ indicate that the interaction occurs in the early phase of aggregation. The solutions are prepared in PBS buffer containing $1 \mathrm{mM}$ EDTA and $1 \mathrm{mM}$ sodium azide at $\mathrm{pH} 7.4$ and incubated at $37^{\circ} \mathrm{C}$ with continuous stirring.

Figure 4: Characterization of the IAPP-DnaK interactions by ensemble and single molecule techniques. A) Ensemble fluorescence anisotropy and B) hydrodynamic radii $\left(\mathrm{R}_{\mathrm{h}}\right)$ from FCS measurements of $400 \mathrm{nM}$ TMR-IAPP in presence of 0 to $5 \mu \mathrm{M}$ DnaK. The symbols represent data. The solid line in $\mathrm{A}$ is fit using a single site model. Equilibrium constant obtained is $2.1 \mu \mathrm{M}$. Heterogeneity of the system is reflected in the fluorescence bursts in the photon count traces (C) and in differences in the autocorrelation data (D) obtained from 10 identical FCS measurements on 400 nM TMR-IAPP $+4 \mu \mathrm{M}$ DnaK sample. Fluorescence bursts (C) indicate oligomers. The solutions are prepared in PBS buffer containing $1 \mathrm{mM}$ EDTA and $1 \mathrm{mM}$ sodium azide at $\mathrm{pH} 7.4$ and incubated at RT.

Figure 5: Characterization of the oligomeric IAPP/TMR-DnaK complexes from photon bursts analysis. Photo count traces (A and C) and autocorrelation data (B and D) from 10 identical measurements from $100 \mathrm{nM}$ TMR-DnaK in absence of IAPP (A and B) or in presence of $20 \mu \mathrm{M}$ IAPP $(\mathrm{C}$ and $\mathrm{D})$. Clearly, both photon counts and autocorrelation data are highly heterogeneous in presence of IAPP. E) comparison of photon counting histograms (PCH) indicate about $8 \%$ of TMR-DnaK is 
partitioned into the bursts. F) histogram of the brightness of the bursts. The solid line is a fit assuming a gaussian distribution. Mean brightness of the bursts $(130 \mathrm{kHz})$ is about 16-times brighter than the monomeric TMR-DnaK. The solutions are prepared in PBS buffer containing $1 \mathrm{mM}$ EDTA and $1 \mathrm{mM}$ sodium azide at $\mathrm{pH} 7.4$ and incubated at RT.

Figure 6: Characterization of IAPP-DnaK complexes following fractionation by Size exclusion chromatography (SEC) or Field flow fractionation (FFF). A) SEC-MALS and C) FFF-MALS of $10 \mu \mathrm{M}$ DnaK in absence and presence of $20 \mu \mathrm{M}$ IAPP. The solid lines indicate refractive index (RI), which is a measure of concentration and empty circles represent molecular weight (MW) measured by MALS for DnaK (black) and DnaK+IAPP (Red) samples. B) $\mathrm{R}_{\mathrm{h}}$ measured using FCS of TMR-IAPP, TMR-DnaK and TMR-IAPP + DnaK following purification using SEC or FFF. Clearly, $\mathrm{R}_{\mathrm{h}}$ of TMRIAPP + DnaK is similar to TMR-DnaK indicating binding of TMR-DnaK to small oligomers of IAPP. The solutions are prepared in PBS buffer containing $1 \mathrm{mM}$ EDTA and $1 \mathrm{mM}$ sodium azide at $\mathrm{pH} 7.4$ and incubated at RT. 


\section{Details of the Supporting Information}

Figure S1. Mass spectrometry of the TMR-IAPP/DnaK sample following fractionation by Size Exclusion Chromatography

Figure S2. Autocorrelation data $\mathrm{G}(\tau)$ obtained from FCS measurements of the SEC or FFF purified TMR-IAPP/DnaK solution

Table S1. Summary of the parameters obtained from fitting of the FCS data

Figure S3. Mass spectrometry of the purified chemically synthesized IAPP

Figure S4. SDS-PAGE of purified E. coli DnaK, MTB-Hsp70 and human Hsc70 


\section{References}

1. Donath, M. Y., Ehses, J. A., Maedler, K., Schumann, D. M., Ellingsgaard, H., Eppler, E., and Reinecke, M. (2005) Mechanisms of $\beta$-Cell Death in Type 2 Diabetes. Diabetes 54, S108S113

2. Höppener, J. W. M., Oosterwijk, C., Nieuwenhuis, M. G., Posthuma, G., Thijssen, J. H. H., Vroom, T. M., Ahrén, B., and Lips, C. J. M. (1999) Extensive islet amyloid formation is induced by development of Type II diabetes mellitus and contributes to its progression: pathogenesis of diabetes in a mouse model. Diabetologia 42, 427-434

3. Hayden, M. R., Tyagi, S. C., Kerklo, M. M., and Nicolls, M. R. (2005) Type 2 diabetes mellitus as a conformational disease. JOP 6, 287-302

4. Jeong, H. R., and An, S. S. (2015) Causative factors for formation of toxic islet amyloid polypeptide oligomer in type 2 diabetes mellitus. Clinical interventions in aging 10, 18731879

5. Yonemoto, I. T., Kroon, G. J., Dyson, H. J., Balch, W. E., and Kelly, J. W. (2008) Amylin proprotein processing generates progressively more amyloidogenic peptides that initially sample the helical state. Biochemistry 47, 9900-9910

6. Meier, J. J., Kayed, R., Lin, C.-Y., Gurlo, T., Haataja, L., Jayasinghe, S., Langen, R., Glabe, C. G., and Butler, P. C. (2006) Inhibition of human IAPP fibril formation does not prevent $\beta$ cell death: evidence for distinct actions of oligomers and fibrils of human IAPP. American Journal of Physiology-Endocrinology and Metabolism 291, E1317-E1324

7. $\quad$ Alfredo Lorenzo, B. R., Gordon C. Weir \& Bruce A. Yankner. (1994) Pancreatic islet cell toxicity of amylin associated with type-2 diabetes mellitus. Nature 368, 756-760

8. J Janson, R. H. A., D Harrison, S McIntyre and P C Butler. (1999) The mechanism of islet amyloid polypeptide toxicity is membrane disruption by intermediate-sized toxic amyloid particles. Diabetes $\mathbf{4 8}, 491-498$

9. Lin, C. Y., Gurlo, T., Kayed, R., Butler, A. E., Haataja, L., Glabe, C. G., and Butler, P. C. (2007) Toxic human islet amyloid polypeptide (h-IAPP) oligomers are intracellular, and vaccination to induce anti-toxic oligomer antibodies does not prevent h-IAPP-induced betacell apoptosis in h-IAPP transgenic mice. Diabetes 56, 1324-1332

10. Balch, W. E. M., R. I. Dillin, A. Kelly, J. W. (2008) Adapting proteostasis for disease intervention. Science (New York, N.Y.) 319, 916-919

11. Hartl, F. U., and Hayer-Hartl, M. (2009) Converging concepts of protein folding in vitro and in vivo. Nature structural \& molecular biology 16, 574-581

12. Mannini, B., and Chiti, F. (2017) Chaperones as Suppressors of Protein Misfolded Oligomer Toxicity. Frontiers in molecular neuroscience 10, 98

13. Atalay, M., Oksala, N., Lappalainen, J., Laaksonen, D. E., Sen, C. K., and Roy, S. (2009) Heat shock proteins in diabetes and wound healing. Current protein \& peptide science 10, $85-$ 95

14. Krause, M., Heck, T. G., Bittencourt, A., Scomazzon, S. P., Newsholme, P., Curi, R., and Homem de Bittencourt, P. I., Jr. (2015) The chaperone balance hypothesis: the importance of the extracellular to intracellular HSP70 ratio to inflammation-driven type 2 diabetes, the effect of exercise, and the implications for clinical management. Mediators of inflammation 2015, 249205

15. Chung, J., Nguyen, A. K., Henstridge, D. C., Holmes, A. G., Chan, M. H., Mesa, J. L., Lancaster, G. I., Southgate, R. J., Bruce, C. R., Duffy, S. J., Horvath, I., Mestril, R., Watt, M. J., Hooper, P. L., Kingwell, B. A., Vigh, L., Hevener, A., and Febbraio, M. A. (2008) HSP72 protects against obesity-induced insulin resistance. Proceedings of the National Academy of Sciences of the United States of America 105, 1739-1744

16. Rosas, P. C., Nagaraja, G. M., Kaur, P., Panossian, A., Wickman, G., Garcia, L. R., AlKhamis, F. A., and Asea, A. A. (2016) Hsp72 (HSPA1A) Prevents Human Islet Amyloid Polypeptide Aggregation and Toxicity: A New Approach for Type 2 Diabetes Treatment. PLOS ONE 11, e0149409 
17. Klucken, J., Shin, Y., Masliah, E., Hyman, B. T., and McLean, P. J. (2004) Hsp70 Reduces alpha-Synuclein Aggregation and Toxicity. The Journal of biological chemistry 279, 2549725502

18. Press, M., Jung, T., Konig, J., Grune, T., and Hohn, A. (2018) Protein aggregates and proteostasis in aging: Amylin and beta-cell function. Mechanisms of ageing and development

19. Kavanagh, K., Flynn, D. M., Jenkins, K. A., Zhang, L., and Wagner, J. D. (2011) Restoring HSP70 deficiencies improves glucose tolerance in diabetic monkeys. American journal of physiology. Endocrinology and metabolism 300, E894-901

20. Bongiovanni, M. N., Aprile, F. A., Sormanni, P., and Vendruscolo, M. (2018) A Rationally Designed Hsp70 Variant Rescues the Aggregation-Associated Toxicity of Human IAPP in Cultured Pancreatic Islet beta-Cells. International journal of molecular sciences 19

21. Evans, C. G., Wisen, S., and Gestwicki, J. E. (2006) Heat shock proteins 70 and 90 inhibit early stages of amyloid beta-(1-42) aggregation in vitro. The Journal of biological chemistry 281, 33182-33191

22. Luk, K. C., Mills, I. P., Trojanowski, J. Q., and Lee, V. M. (2008) Interactions between Hsp70 and the hydrophobic core of alpha-synuclein inhibit fibril assembly. Biochemistry 47, 12614-12625

23. Wacker, J. L., Zareie, M. H., Fong, H., Sarikaya, M., and Muchowski, P. J. (2004) Hsp70 and Hsp40 attenuate formation of spherical and annular polyglutamine oligomers by partitioning monomer. Nature structural \& molecular biology 11, 1215-1222

24. Baughman, H. E. R., Clouser, A. F., Klevit, R. E., and Nath, A. (2018) HspB1 and Hsc70 chaperones engage distinct tau species and have different inhibitory effects on amyloid formation. The Journal of biological chemistry 293, 2687-2700

25. Chien, V., Aitken, J. F., Zhang, S., Buchanan, C. M., Hickey, A., Brittain, T., Cooper, G. J., and Loomes, K. M. (2010) The chaperone proteins HSP70, HSP40/DnaJ and GRP78/BiP suppress misfolding and formation of beta-sheet-containing aggregates by human amylin: a potential role for defective chaperone biology in Type 2 diabetes. The Biochemical journal 432, 113-121

26. Gao, X., Carroni, M., Nussbaum-Krammer, C., Mogk, A., Nillegoda, N. B., Szlachcic, A., Guilbride, D. L., Saibil, H. R., Mayer, M. P., and Bukau, B. (2015) Human Hsp70 Disaggregase Reverses Parkinson's-Linked alpha-Synuclein Amyloid Fibrils. Molecular cell 59, 781-793

27. Repalli, J., and Meruelo, D. (2015) Screening strategies to identify HSP70 modulators to treat Alzheimer's disease. Drug design, development and therapy 9, 321-331

28. Muchowski, P. J., Schaffar, G., Sittler, A., Wanker, E. E., Hayer-Hartl, M. K., and Hartl, F. U. (2000) Hsp70 and hsp40 chaperones can inhibit self-assembly of polyglutamine proteins into amyloid-like fibrils. Proceedings of the National Academy of Sciences of the United States of America 97, 7841-7846

29. Sakono, M., and Kidani, T. (2017) ATP-independent inhibition of amyloid beta fibrillation by the endoplasmic reticulum resident molecular chaperone GRP78. Biochemical and biophysical research communications $\mathbf{4 9 3}, 500-503$

30. Kundel, F., De, S., Flagmeier, P., Horrocks, M. H., Kjaergaard, M., Shammas, S. L., Jackson, S. E., Dobson, C. M., and Klenerman, D. (2018) Hsp70 Inhibits the Nucleation and Elongation of Tau and Sequesters Tau Aggregates with High Affinity. ACS Chem Biol 13, 636-646

31. Schonfeld, H. J., Schmidt, D., Schroder, H., and Bukau, B. (1995) The DnaK chaperone system of Escherichia coli: quaternary structures and interactions of the DnaK and GrpE components. The Journal of biological chemistry 270, 2183-2189

32. Garai, K., and Frieden, C. (2013) Quantitative analysis of the time course of Abeta oligomerization and subsequent growth steps using tetramethylrhodamine-labeled Abeta. Proceedings of the National Academy of Sciences of the United States of America 110, 33213326

33. Zuiderweg, E. R. P., Hightower, L. E., and Gestwicki, J. E. (2017) The remarkable multivalency of the Hsp70 chaperones. Cell Stress Chaperones 22, 173-189 
34. Whiten, D. R., Cox, D., Horrocks, M. H., Taylor, C. G., De, S., Flagmeier, P., Tosatto, L., Kumita, J. R., Ecroyd, H., Dobson, C. M., Klenerman, D., and Wilson, M. R. (2018) SingleMolecule Characterization of the Interactions between Extracellular Chaperones and Toxic $\alpha$ Synuclein Oligomers. Cell reports 23, 3492-3500

35. Sil, T. B., Sahoo, B., Bera, S. C., and Garai, K. (2018) Quantitative Characterization of Metastability and Heterogeneity of Amyloid Aggregates. Biophysical journal 114, 800-811

36. Scales, N., and Swain, P. S. (2020) Resolving fluorescent species by their brightness and diffusion using correlated photon-counting histograms. PLOS ONE 14, e0226063

37. Sahoo, B., Sil, T. B., Karmakar, B., and Garai, K. (2018) A Fluorescence Correlation Spectrometer for Measurements in Cuvettes. Biophysical journal 115, 455-466

38. Hubin, E., Verghese, P. B., van Nuland, N., and Broersen, K. (2019) Apolipoprotein E associated with reconstituted high-density lipoprotein-like particles is protected from aggregation. FEBS letters 593, 1144-1153

39. Richter, K., Haslbeck, M., and Buchner, J. (2010) The heat shock response: life on the verge of death. Molecular cell 40, 253-266

40. Mannini, B., Cascella, R., Zampagni, M., van Waarde-Verhagen, M., Meehan, S., Roodveldt, C., Campioni, S., Boninsegna, M., Penco, A., Relini, A., Kampinga, H. H., Dobson, C. M., Wilson, M. R., Cecchi, C., and Chiti, F. (2012) Molecular mechanisms used by chaperones to reduce the toxicity of aberrant protein oligomers. Proceedings of the National Academy of Sciences of the United States of America 109, 12479-12484

41. Clerico, E. M., Tilitsky, J. M., Meng, W., and Gierasch, L. M. (2015) How hsp70 molecular machines interact with their substrates to mediate diverse physiological functions. Journal of molecular biology 427, 1575-1588

42. $\quad$ Fernandez-Funez, P., Sanchez-Garcia, J., de Mena, L., Zhang, Y., Levites, Y., Khare, S., Golde, T. E., and Rincon-Limas, D. E. (2016) Holdase activity of secreted Hsp70 masks amyloid-beta42 neurotoxicity in Drosophila. Proceedings of the National Academy of Sciences of the United States of America 113, E5212-5221

43. Horvath, I., Blockhuys, S., Sulskis, D., Holgersson, S., Kumar, R., Burmann, B. M., and Wittung-Stafshede, P. (2019) Interaction between Copper Chaperone Atox 1 and Parkinson's Disease Protein $\alpha$-Synuclein Includes Metal-Binding Sites and Occurs in Living Cells. ACS chemical neuroscience 10, 4659-4668

44. Shorter, J. (2011) The mammalian disaggregase machinery: Hsp110 synergizes with Hsp70 and Hsp40 to catalyze protein disaggregation and reactivation in a cell-free system. PLoS ONE 6, e26319

45. Zietkiewicz, S., Lewandowska, A., Stocki, P., and Liberek, K. (2006) Hsp70 chaperone machine remodels protein aggregates at the initial step of Hsp70-Hsp100-dependent disaggregation. The Journal of biological chemistry 281, 7022-7029

46. Rosenzweig, R., Sekhar, A., Nagesh, J., and Kay, L. E. (2017) Promiscuous binding by Hsp70 results in conformational heterogeneity and fuzzy chaperone-substrate ensembles. eLife 6

47. Fourie, A. M., Sambrook, J. F., and Gething, M. J. (1994) Common and divergent peptide binding specificities of hsp70 molecular chaperones. The Journal of biological chemistry $\mathbf{2 6 9}$, 30470-30478

48. Flynn, G. C., Chappell, T. G., and Rothman, J. E. (1989) Peptide binding and release by proteins implicated as catalysts of protein assembly. Science (New York, N.Y.) 245, 385-390

49. Schröder, H., Langer, T., Hartl, F. U., and Bukau, B. (1993) DnaK, DnaJ and GrpE form a cellular chaperone machinery capable of repairing heat-induced protein damage. The EMBO journal 12, 4137-4144

50. Van Durme, J., Maurer-Stroh, S., Gallardo, R., Wilkinson, H., Rousseau, F., and Schymkowitz, J. (2009) Accurate prediction of DnaK-peptide binding via homology modelling and experimental data. PLoS computational biology 5, e1000475

51. Ghosh, S., Sil, T. B., Dolai, S., and Garai, K. (2019) High-affinity multivalent interactions between apolipoprotein $\mathrm{E}$ and the oligomers of amyloid- $\beta$. The FEBS journal 286, 4737-4753

52. Kampinga, H. H., and Craig, E. A. (2010) The HSP70 chaperone machinery: J proteins as drivers of functional specificity. Nat Rev Mol Cell Biol 11, 579-592 
bioRxiv preprint doi: https://doi.org/10.1101/2020.03.30.016881; this version posted March 31, 2020. The copyright holder for this preprint (which was not certified by peer review) is the author/funder, who has granted bioRxiv a license to display the preprint in perpetuity. It is made available under aCC-BY-NC-ND 4.0 International license.

53. Li, H., Zhu, H., Sarbeng, E. B., Liu, Q., Tian, X., Yang, Y., Lyons, C., Zhou, L., and Liu, Q. (2020) An unexpected second binding site for polypeptide substrates is essential for Hsp70 chaperone activity. The Journal of biological chemistry 295, 584-596

54. Kellner, R., Hofmann, H., Barducci, A., Wunderlich, B., Nettels, D., and Schuler, B. (2014) Single-molecule spectroscopy reveals chaperone-mediated expansion of substrate protein. Proceedings of the National Academy of Sciences of the United States of America 111, 1335513360

55. Kettern, N., Dreiseidler, M., Tawo, R., and Höhfeld, J. (2010) Chaperone-assisted degradation: multiple paths to destruction. Biol Chem 391, 481-489

56. Mangione, M. R., Vilasi, S., Marino, C., Librizzi, F., Canale, C., Spigolon, D., Bucchieri, F., Fucarino, A., Passantino, R., Cappello, F., Bulone, D., and San Biagio, P. L. (2016) Hsp60, amateur chaperone in amyloid-beta fibrillogenesis. Biochimica et biophysica acta 1860, 2474-2483

57. Marek, P., Woys, A. M., Sutton, K., Zanni, M. T., and Raleigh, D. P. (2010) Efficient microwave-assisted synthesis of human islet amyloid polypeptide designed to facilitate the specific incorporation of labeled amino acids. Organic letters 12, 4848-4851

58. Montgomery, D. L., Morimoto, R. I., and Gierasch, L. M. (1999) Mutations in the substrate binding domain of the Escherichia coli $70 \mathrm{kDa}$ molecular chaperone, DnaK, which alter substrate affinity or interdomain coupling. Journal of molecular biology 286, 915-932

59. Swain, J. F., Schulz, E. G., and Gierasch, L. M. (2006) Direct comparison of a stable isolated Hsp70 substrate-binding domain in the empty and substrate-bound states. The Journal of biological chemistry 281, 1605-1611

60. Puchalla, J., Krantz, K., Austin, R., and Rye, H. (2008) Burst analysis spectroscopy: a versatile single-particle approach for studying distributions of protein aggregates and fluorescent assemblies. Proceedings of the National Academy of Sciences of the United States of America 105, 14400-14405 

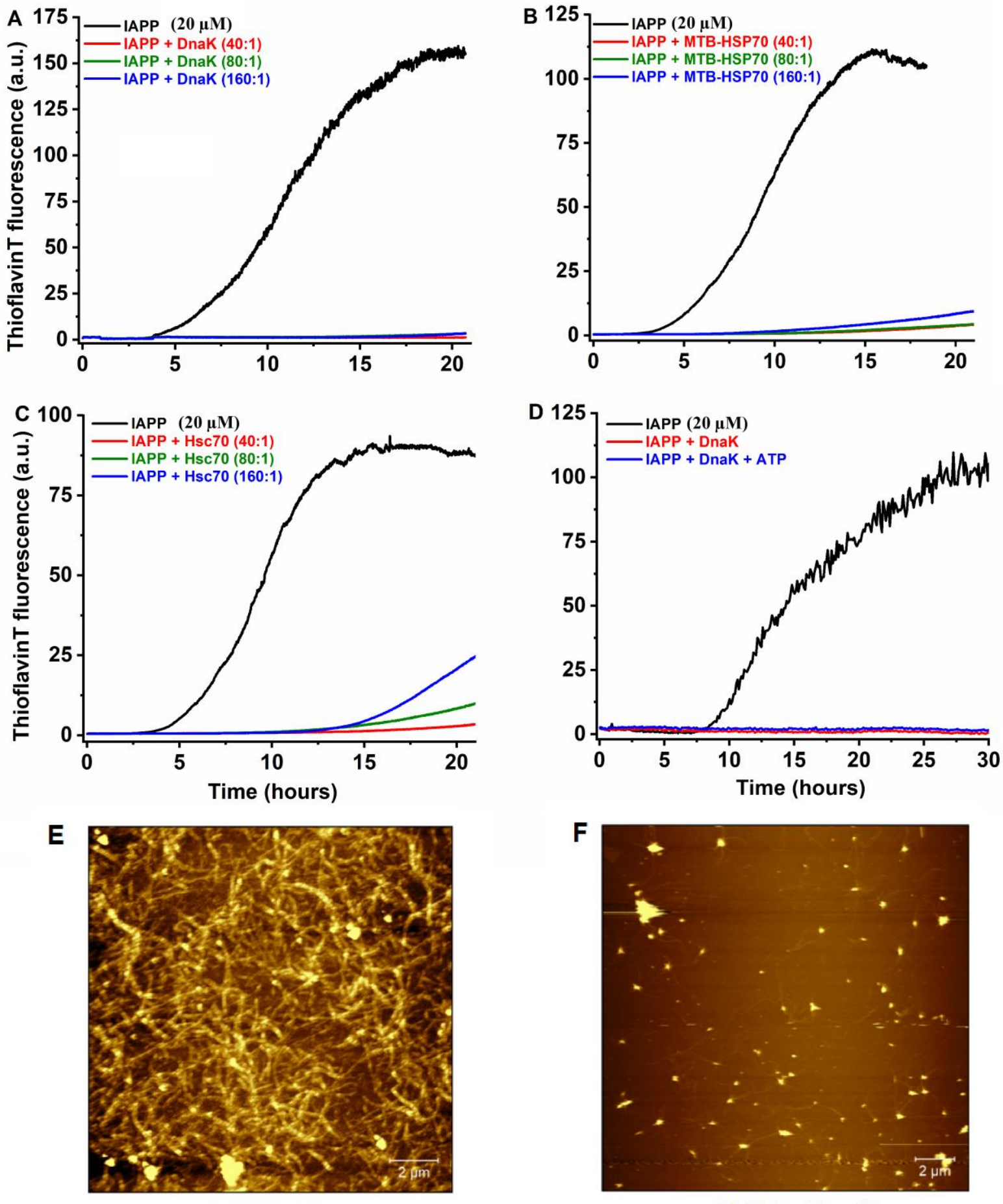

IAPP alone

IAPP + DnaK

Figure 1 

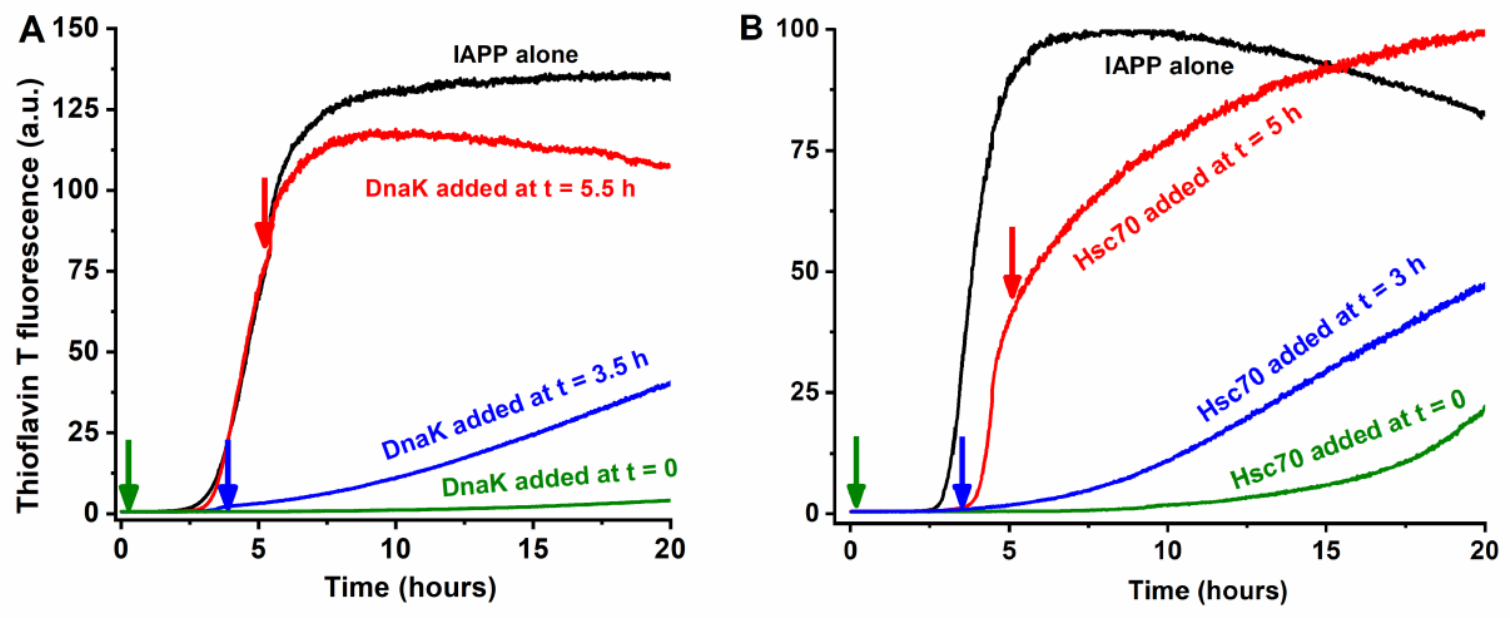

Figure 2 

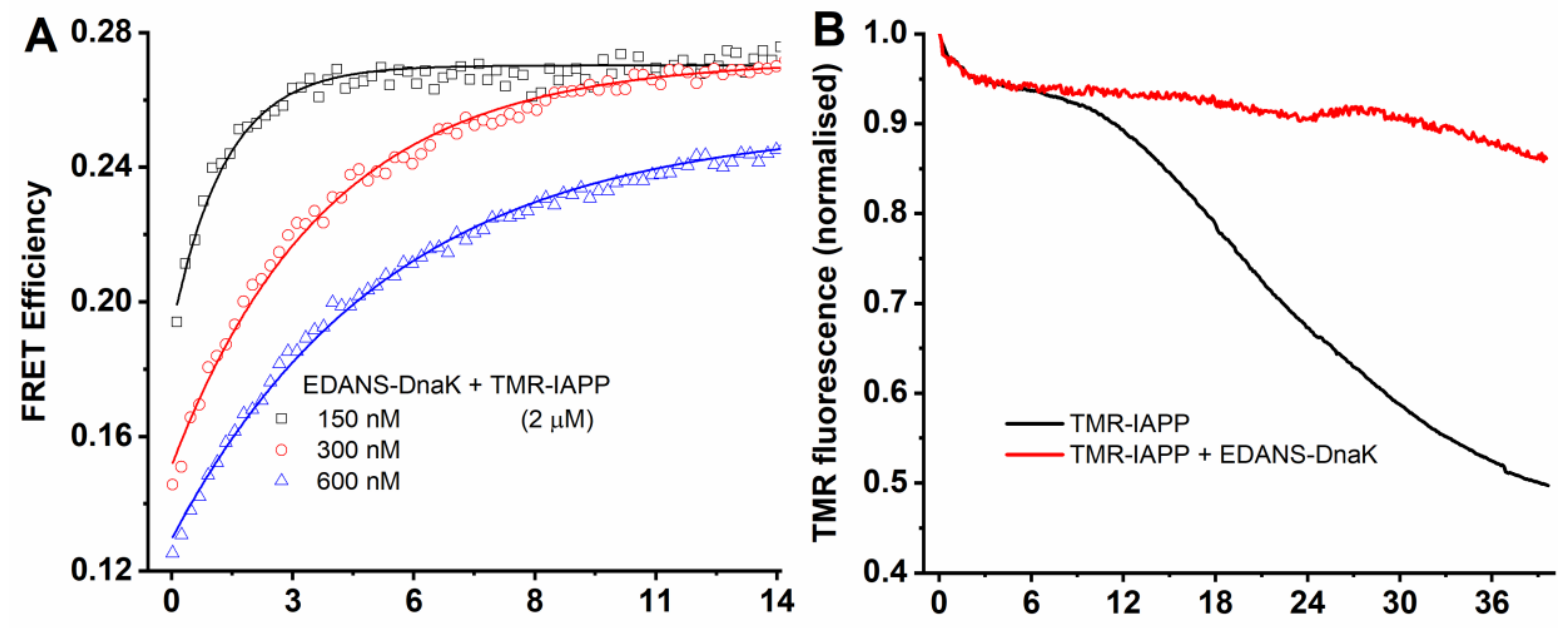

Time (hours)

Figure 3 

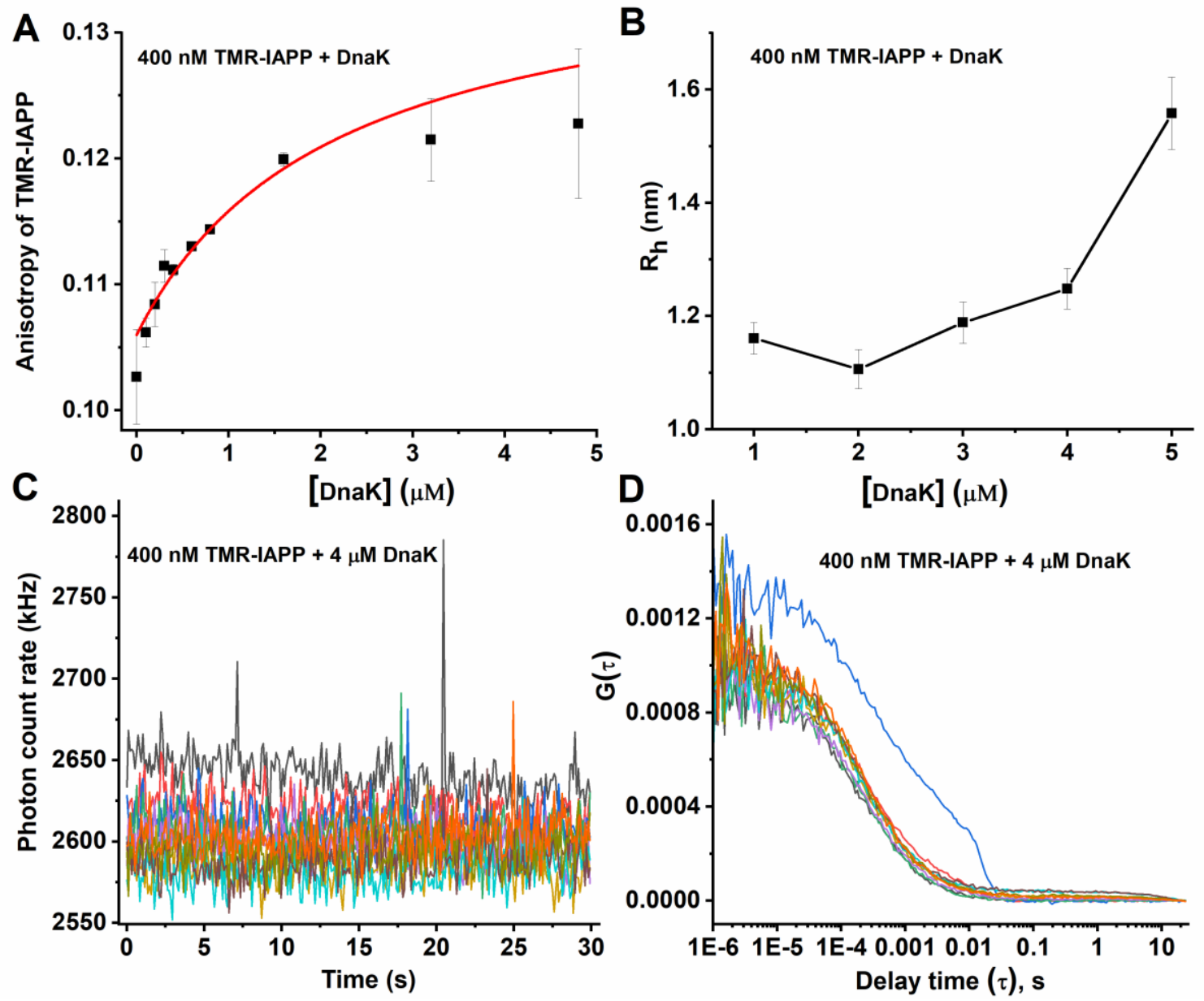

Figure 4 

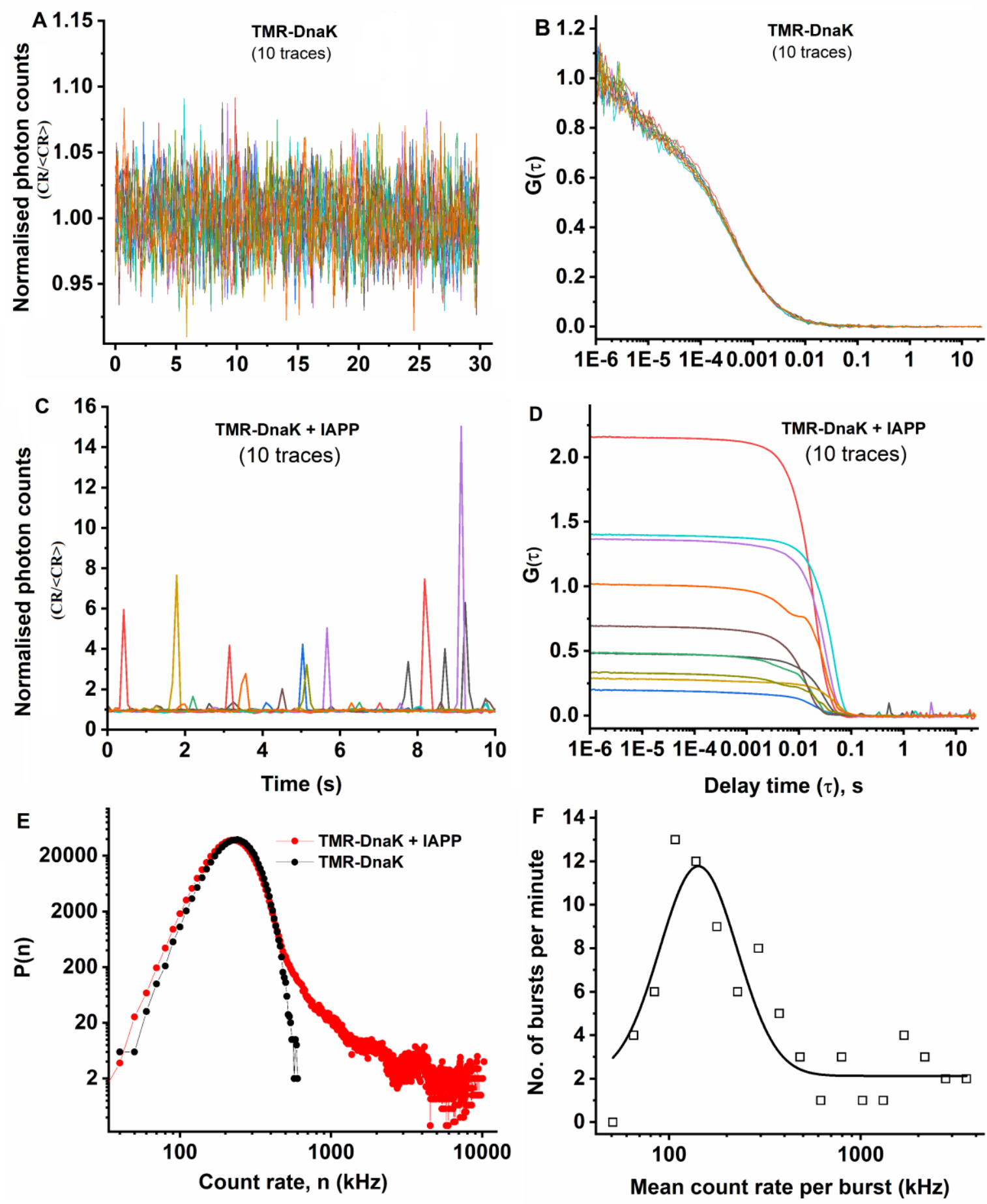

Figure 5 

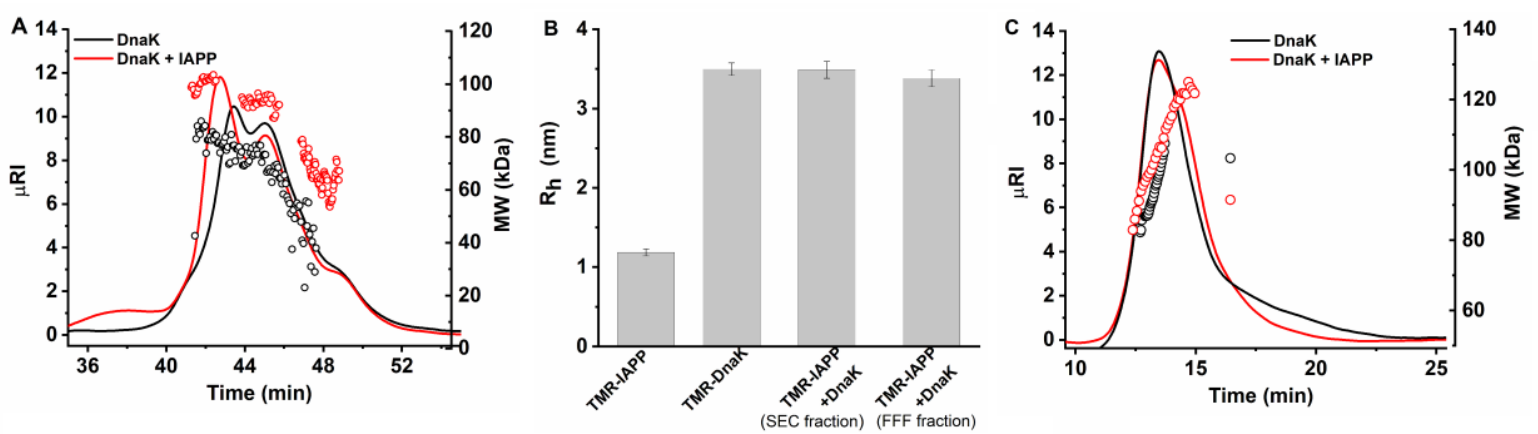

Figure 6 


\section{Supporting information}

Hsp70 inhibits aggregation of Islet amyloid polypeptide by binding to the heterogeneous prenucleation oligomers

Neeraja Chilukoti ${ }^{1,2}$, Bankanidhi Sahoo ${ }^{1,3}$, Deepa $\mathrm{S}^{1}$, Sreelakshmi Cherakara ${ }^{1}$, Mithun Maddheshiya ${ }^{1}$ and Kanchan Garai ${ }^{1, *}$

${ }^{1}$ Tata Institute of Fundamental Research, 36/P Gopanpally, Serilingampally, Hyderabad 500019, India

${ }^{2}$ Current address: Centre for Cellular \& Molecular Biology, Habsiguda, Hyderabad - 500007

${ }^{3}$ Current address: Dr. Reddy's Laboratories, Bachupally, Hyderabad 500090, India

Running title- Biophysical investigation of Hsp70-IAPP interactions

"Towhom correspondence should be addressed: kanchan@tifrh.res.in

\section{List of Supplementary Figures and Tables}

Figure S1. Mass spectrometry of the TMR-IAPP/DnaK sample following fractionation by Size Exclusion Chromatography

Figure S2. Autocorrelation data $\mathrm{G}(\tau)$ obtained from FCS measurements of the SEC or FFF purified TMR-IAPP/DnaK solution

Table S1. Summary of the parameters obtained from fitting of the FCS data

Figure S3. Mass spectrometry of the purified chemically synthesized IAPP

Figure S4. SDS-PAGE of purified E. coli DnaK, MTB-Hsp70 and human Hsc70 


\section{Mass spectrometry of the TMR-IAPP/DnaK solution following fractionation by Size exclusion}

\section{Chromatography}

TMR-IAPP/DnaK complexes were fractionated using size exclusion chromatography (SEC), which is coupled to the multiangle light scattering (MALS) setup. The fraction exhibiting the highest peak in the refractive index (RI) detector was collected for mass spectrometry measurements. Mass spectrometry measurements were performed using a 6545 Q-TOF ESI-MS (Agilent technologies). The spectrometer is coupled to a HPLC for desalting of the injected sample. The $\mathrm{m} / \mathrm{z}$ spectra were deconvoluted with the MassHunter software (Agilent, UK) using the resolved isotope deconvolution method for measuring the mass of peptides (MW $<10 \mathrm{kDa}$ ), and the maximum entropy deconvolution method for measuring the mass of proteins $(\mathrm{MW}>10 \mathrm{kDa})$.
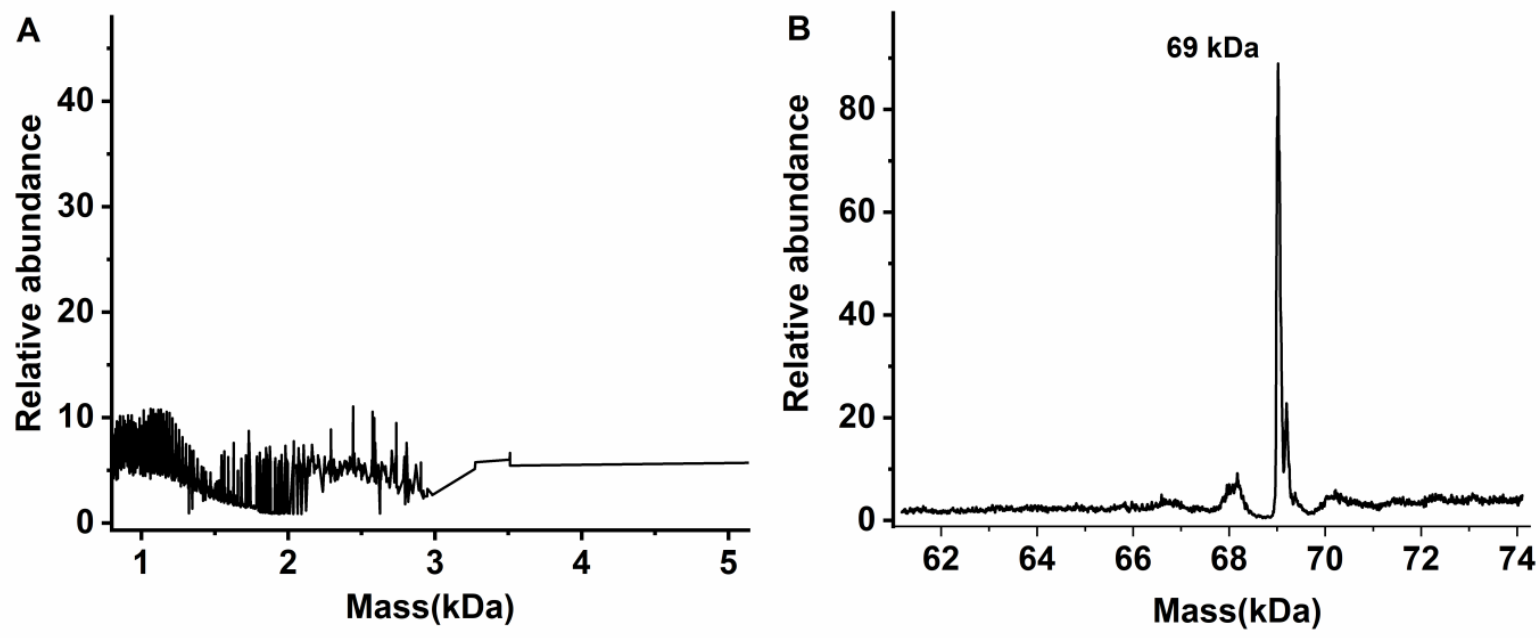

Supplementary Figure S1: Mass spectrometry of the TMR-IAPP/DnaK sample following fractionation by SEC. A) Deconvolution of the $\mathrm{m} / \mathrm{z}$ spectra of IAPP was performed within a mass range of 0 to $5000 \mathrm{Da}$ using the resolved isotope method. Deconvolution within this mass range did not detect any IAPP, indicating that concentration of IAPP in the SEC fraction must be very low. B) Detection of DnaK by deconvolution of $\mathrm{m} / \mathrm{z}$ spectrum within a mass range of 60 to $80 \mathrm{kDa}$. Presence of DnaK (69 kDa) is quite clear. Hence, the SEC purified fraction contains primarily free DnaK and possibly a very low concentration of the complexes. 


\section{Autocorrelation data G( $\tau)$ obtained from the FCS measurements of SEC or FFF purified TMR-} IAPP/DnaK

FCS measurements were performed on free rhodamine B, TMR-IAPP, TMR-DnaK and SEC or FFF purified fractions of TMR-IAPP/DnaK solution. Free rhodamine B (50 nM) was used as a control to characterize the FCS observation volume. TMR-IAPP (50 $\mathrm{nM})$ and TMR-DnaK $(50 \mathrm{nM})$ were used to estimate the hydrodynamic radii $\left(\mathrm{R}_{\mathrm{h}}\right)$ of these proteins in the unbound monomeric forms. The major fractions of the SEC or FFF purified TMR-IAPP/DnaK solution was used directly for FCS measurements. The FCS data were analysed using a single diffusion and single relaxation model (1) (see supplementary eq. S1). The relaxation component was used to take care of the contributions from conformational fluctuations and/or the triplet state dynamics. Concentrations of the purified samples were determined from the $\mathrm{G}(0)$ of the autocorrelation data..

$$
G(\tau)=\frac{1}{<N>}\left(\frac{1-F_{R}+F_{R} \operatorname{Exp}\left(-\frac{\tau}{\tau_{R}}\right)}{1-F_{R}}\right) \frac{1}{\left(1+\frac{\tau}{\tau_{D}}\right)\left(1+\frac{\tau}{\omega^{2} \tau_{D}}\right)^{0.5}}
$$

Here $\langle\mathrm{N}\rangle$ is average number of molecules in the FCS observation volume. $\mathrm{F}_{\mathrm{R}}$ and $\tau_{\mathrm{R}}$ are the relative amplitude and the characteristic time of the relaxation component respectively. The $\tau_{\mathrm{D}}$ is the diffusion time of the molecule and $\omega$ is the axial ratio of the FCS observation volume. It may noted here that $\langle\mathrm{N}\rangle^{-1}$ is $\mathrm{G}(0) \times\left(1-\mathrm{F}_{\mathrm{R}}\right)$. Hence, in absence of the relaxation component $\langle\mathrm{N}\rangle^{-1}$ is simply equal to $\mathrm{G}(0)$. Hydrodynamic radius $\left(\mathrm{R}_{\mathrm{h}}\right)$ of the proteins are estimated from the measured diffusion times using the following relationship (see eq. $\mathrm{S} 2$ ). The $\mathrm{R}_{\mathrm{h}}$ of rhodamine B used is $0.57 \mathrm{~nm}$ (2).

$$
R_{h, \text { protein }}=\frac{\tau_{D, \text { protein }}}{\tau_{D, \text { rhodamineB }}} R_{h, \text { rhodamineB }}
$$




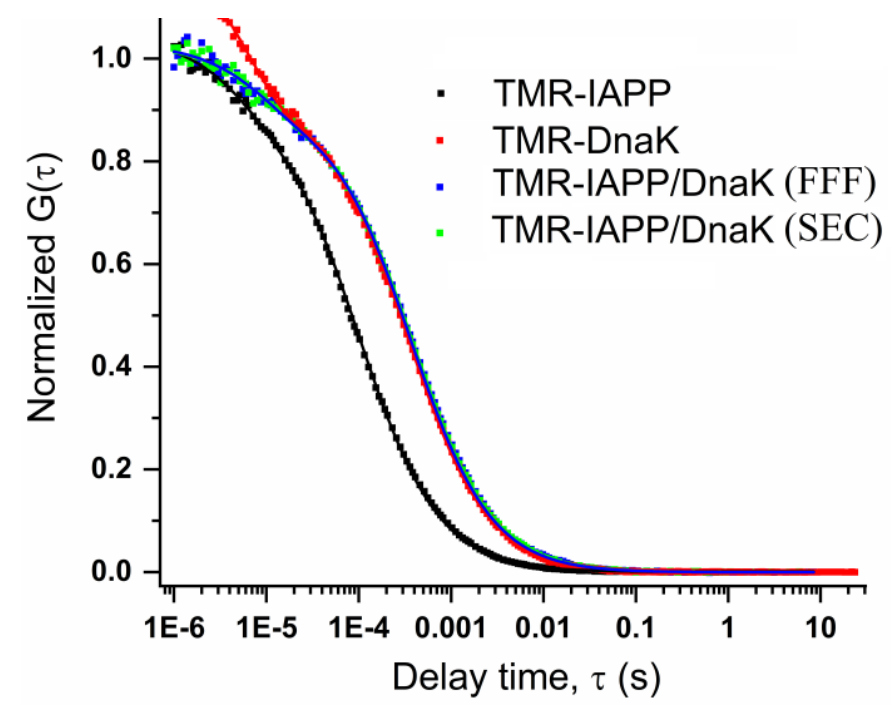

Supplementary Figure S2: Normalized autocorrelation data $G(\tau)$ obtained from FCS measurements of SEC or FFF purified IAPP/DnaK. TMR-IAPP (black), TMR-DnaK (red), SEC purified fraction (green) or FFF purified fraction (blue) of the TMR-IAPP/DnaK sample in PBS, pH 7.4 buffer at RT. For SEC or FFF purified samples, the fractions exhibiting the highest RI were taken for the FCS measurements. The symbols represent data and the solid lines were fit using a single diffusion and single relaxation model (see supplementary eq. S1). The diffusion time $\left(\tau_{\mathrm{D}}\right)$ was used for estimation of the hydrodynamic radius of the molecules (see supplementary eq. S2).

Supplementary Table S1: Summary of the parameters obtained from fitting of the FCS data

\begin{tabular}{lllllll}
\hline Sample & $\langle N\rangle$ & $\tau_{\mathrm{D}}(\mu \mathrm{s})$ & $F$ & $\tau_{\mathrm{R}}(\mu \mathrm{s})$ & Conc. $(\mathrm{nM})$ & $\mathrm{R}_{\mathrm{h}}(\mathrm{nm})$ \\
\hline TMR-IAPP & $22 \pm 2$ & $119 \pm 2$ & 0 (fixed) & NA & 20 & 1.2 \\
TMR-DnaK & $55 \pm 2$ & $398 \pm 4$ & $0.2 \pm 0.001$ & $14 \pm 1$ & 50 & 3.3 \\
$\begin{array}{l}\text { TMR-IAPP/DnaK } \\
\text { (from FFF) }\end{array}$ & $16 \pm 3$ & $397 \pm 20$ & $0.1 \pm 0.004$ & $15 \pm 1$ & 15 & 3.3 \\
TMR-IAPP/DnaK & & & & & & \\
(from SEC) & $50 \pm 5$ & $385 \pm 20$ & $0.09 \pm 0.003$ & $15 \pm 1$ & 45 & 3.3
\end{tabular}

The abbreviations $\langle\mathrm{N}\rangle, \tau_{\mathrm{D}}, \mathrm{F}$ and $\tau_{\mathrm{R}}$ have usual meaning as described in supplementary eq. $\mathrm{S} 1$. Concentration is calculated from $\langle\mathrm{N}\rangle$. In our setup we find $\langle\mathrm{N}\rangle /$ Conc. $=1.1 / \mathrm{nM}$. Calibration of the FCS observation volume is performed using rhodamine B solutions (for details see Ref. 2). The hydrodynamic radius $\left(\mathrm{R}_{\mathrm{h}}\right)$ is estimated using supplementary eq. S2.

*In case of TMR-IAPP the value to $F$ was set to 0.0 to avoid redundancy in the values of the fitting parameters.

Mass spectrometry of chemically synthesized purified IAPP 
A $100 \mu 1$ of IAPP sample was injected into the HPLC fitted with reverse phase column (Advance Biopeptide map column, Agilent), which is connected inline with an ESI-MS mass spectrometer (Agilent technologies, UK). The $\mathrm{m} / \mathrm{z}$ spectra were deconvoluted using the inbuilt program in the resolved isotope mode and mass of $3904.9 \mathrm{Da}$ was noted for IAPP which is similar to that of the theoretical MW of IAPP containing disulphide bridge between 2 and 7 cysteine.

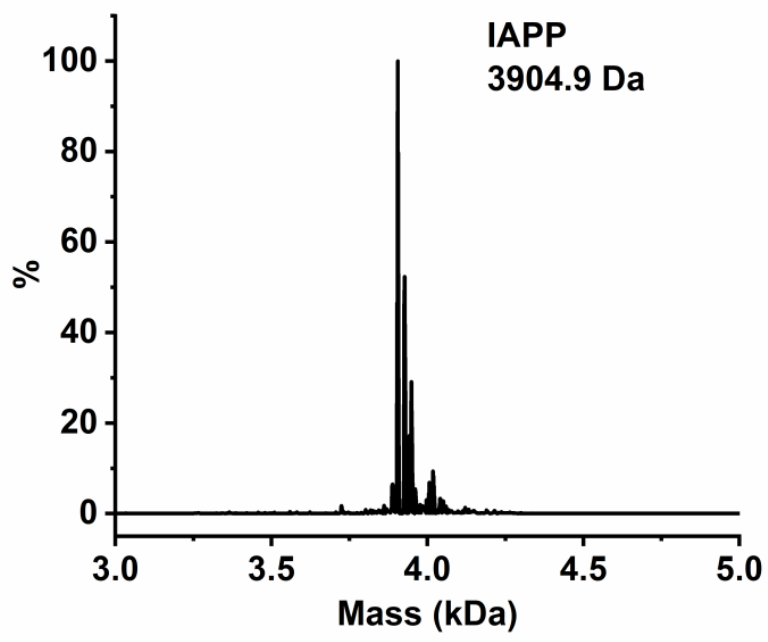

Supplementary Figure S3: Mass spectrometry of chemically synthesized purified IAPP. Molecular weight (MW) and the purity of the chemically synthesized IAPP were confirmed by mass spectrometry. The MW of IAPP was measured to be $3904.9 \mathrm{Da}$, which is same as the theoretical MW of IAPP containing disulphide bridge between cysteines placed at position 2 and 7. MW of the peaks placed on the right side of the main peak are 3925.2 and 3949.2 Da. These peaks are corresponding to the sodium adducts of IAPP. 


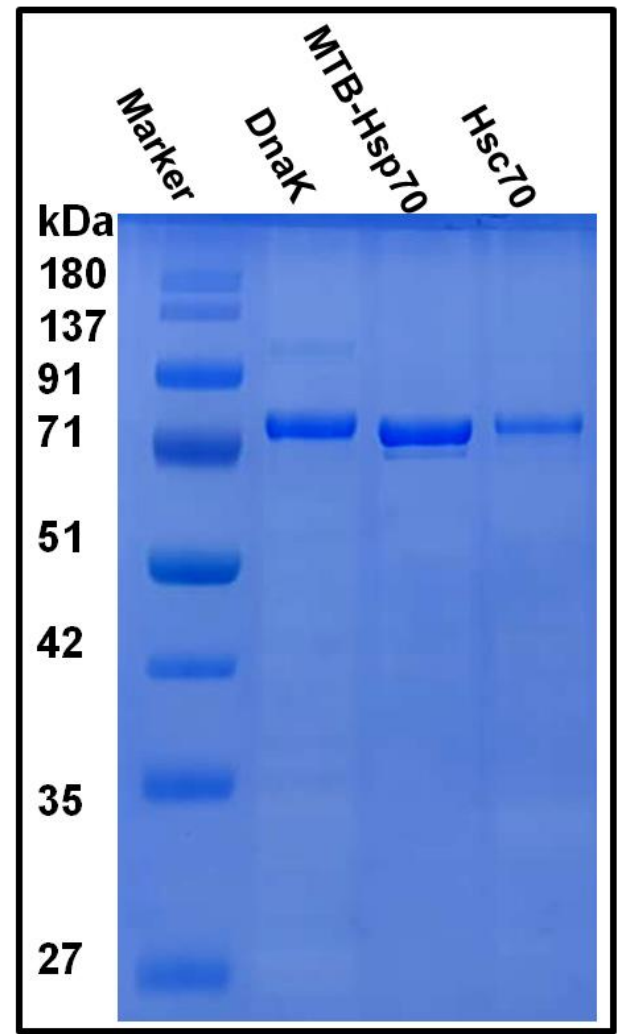

Supplementary Figure S4: SDS-PAGE of purified E. coli DnaK, MTB-Hsp70 and human Hsc70. A single dominant band indicates that the proteins are considerably pure.

\section{References}

1. Sil, T. B., Sahoo, B., and Garai, K. (2018) Building, Characterization, and Applications of Cuvette-FCS in Denaturant-Induced Expansion of Globular and Disordered Proteins. Methods in enzymology 611, 383-421

2. Sahoo, B., Sil, T. B., Karmakar, B., and Garai, K. (2018) A Fluorescence Correlation Spectrometer for Measurements in Cuvettes. Biophysical journal 115, 455-466 